\title{
Analyzing the Impact of Wireless Multi-Hop Networking On Vehicular Safety
}

Rudhir Upretee

West Virginia University

Follow this and additional works at: https://researchrepository.wvu.edu/etd

\section{Recommended Citation}

Upretee, Rudhir, "Analyzing the Impact of Wireless Multi-Hop Networking On Vehicular Safety" (2013). Graduate Theses, Dissertations, and Problem Reports. 654.

https://researchrepository.wvu.edu/etd/654

This Thesis is protected by copyright and/or related rights. It has been brought to you by the The Research Repository @ WVU with permission from the rights-holder(s). You are free to use this Thesis in any way that is permitted by the copyright and related rights legislation that applies to your use. For other uses you must obtain permission from the rights-holder(s) directly, unless additional rights are indicated by a Creative Commons license in the record and/ or on the work itself. This Thesis has been accepted for inclusion in WVU Graduate Theses, Dissertations, and Problem Reports collection by an authorized administrator of The Research Repository @ WVU. For more information, please contact researchrepository@mail.wvu.edu. 


\title{
Analyzing the Impact of Wireless Multi- Hop Networking On Vehicular Safety
}

by

\author{
Rudhir Upretee
}

Thesis submitted to the

Benjamin M. Statler College of Engineering and Mineral Resources at West Virginia University in partial fulfillment of the requirements

for the degree of

Master of Science in

Computer Science

Vinod K. Kulathumani, Ph.D., Chair

Yaser P.Fallah, Ph.D.

Tim Menzies, Ph.D.

Lane Department of Computer Science and Electrical Engineering

Morgantown, West Virginia

2013

Keywords: VCAST, Multi-hop networking, Simulation, Vehicle Safety, Traffic String Stability

Copyright 2013 Rudhir Upretee 


\author{
Abstract \\ Analyzing The Impact Of Wireless Multi-Hop Network On Vehicular Safety \\ by \\ Rudhir Upretee \\ Master of Science in Computer Science \\ West Virginia University \\ Vinod K. Kulathumani, Ph.D., Chair
}

One of the core challenges of Intelligent Transportation System is the dissemination of timely and accurate vehicle information (e.g. speed, position) to geographically large distances without compromising data supply rates from immediate neighbors. This feature is critical for the design of vehicle safety and navigation applications. Single hop broadcasting is often inadequate to ensure vehicle safety when the platoon size is arbitrarily large due to its upper bound on rate and range of wireless message transmission. Existing wireless multi-hop protocols do not ensure reliable message delivery while avoiding network congestion in the shared channel. In this thesis, we make two separate but related investigations to address this challenge - (1) Analyze the impact of distance sensitive multi-hop broadcasting in realistic traffic network (2) Analyze the impact of wireless multi-hop network in vehicle safety. For investigating the first part, we used VCAST, a distance sensitive information propagation technique, in which information is forwarded at a rate that decreases linearly with distance from the source. VCAST is evaluated by using extensive simulations in ns-3, a discrete event simulator for wireless and mobile ad-hoc networks, under different density, source broadcast rates and communication range. To simulate realistic traffic movement, we used $2 \mathrm{~d}$ grids of different sizes and used both uniform and non-uniform mobility. The results show that VCAST is scalable for - large number of vehicles and large source broadcast rates. It is further shown that successful scaling is achieved by reduced number of vehicle records transmitted per second per vehicle for varying network sizes and varying source broadcast rates. Vehicle safety messages for VCAST are piggy backed on heart beat messages and does not require any modifications to the existing vehicular communication standards. For investigating the second part, we implemented a realistic car following model and used string stability analysis as a metric for measuring vehicle safety. The basic idea is to exploit the small network propagation time in disseminating safety messages over large distances, instead of relying on just the predecessor vehicle's state. This enables distant vehicles in a traffic stream to plan well in advance against rear end collisions which could lead to string instability. We also proposed one such proactive method of planning - and that is by controlling the headway time. Through extensive simulations, we obtained results for vehicle safety when some incident is detected abruptly on its course. The results show that proactive planning using multi-hop network makes the entire platoon string stable in the presence of emergency road incidents. 


\section{Acknowledgments}

This thesis would not have been possible without the unrelenting guidance of my committee chair and advisor Dr. Vinod K. Kulathumani. Valuable suggestions and insights from Dr. Vinod helped me to improve the presentation style, correct many mistakes and make the thesis more readable. I am also thankful to Dr. Vinod for providing me the opportunity to work with him. It has been an overwhelming experience and I have learnt a lot both academically and personally by interacting with him.

Dr. Yaser Fallah also deserves a lot of credit. The foundation of my research is based on the classes taught by Dr. Fallah. I am also very grateful to Dr. Tim Menzies for teaching me how to look at data and interpret data. I am thankful to both of them for their support and time throughout the program.

Many thanks to my research group members Rahul Kavi and Goutham Ranganath for their encouragement and support. They played their part in making the work environment fun, challenging and interesting. I would also like to thank all those who directly or indirectly supported me throughout this study.

This work would be incomplete without the continued support and trust from my mom, dad and my elder brother. Thank you for believing in me. I am always indebted to them. 


\section{Contents}

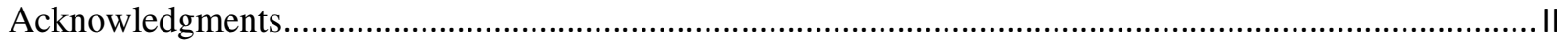

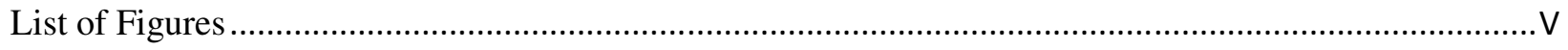

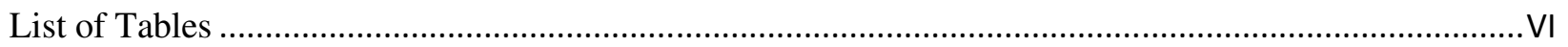

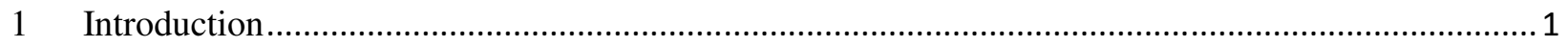

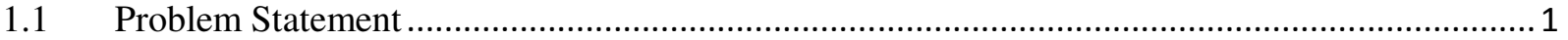

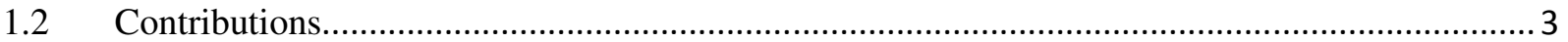

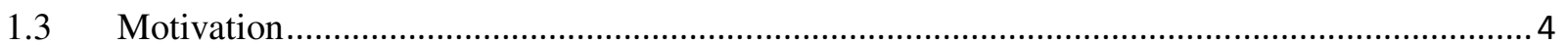

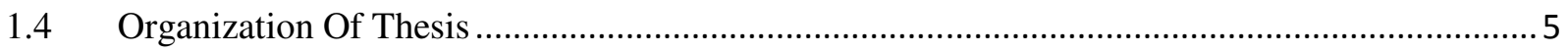

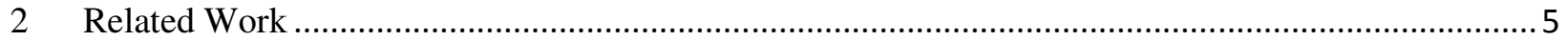

2.1 Distance Sensitive Wireless Multi-Hop Network For VANET …........................................... 5

2.2 Multi-Hop Network In Vehicular Safety Analysis .......................................................... 6

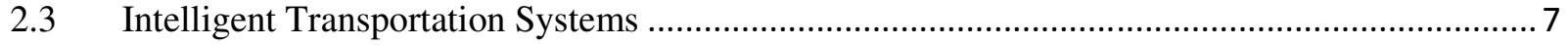

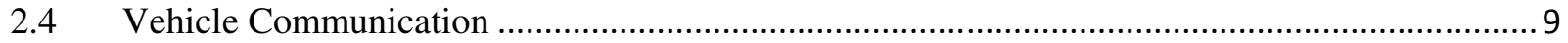

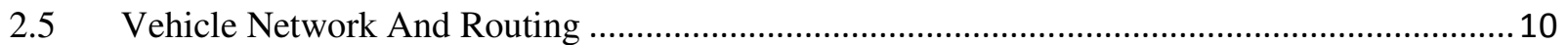

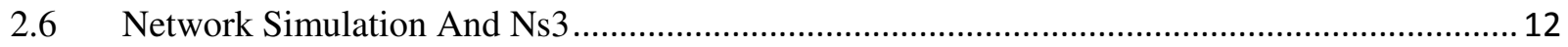

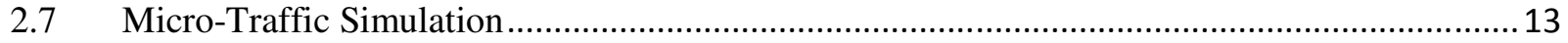

3 Analyzing the Performance and Scalability of VCAST, a distance sensitive multi-hop broadcast

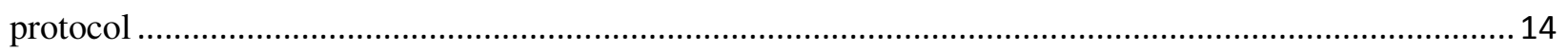

3.1 Distance Sensitive Broadcast Algorithm ....................................................................... 15

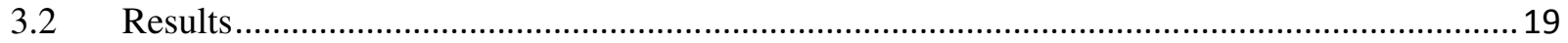

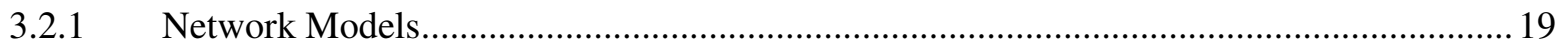

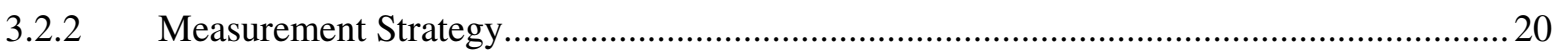

4 Analyzing The Impact of VCAST On Vehicular Safety ............................................................. 25

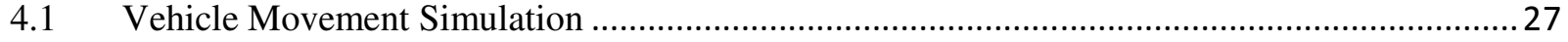

4.1.1 General Car Following Model …............................................................................ 28

4.1.2 Car Following Model With First Order Dynamics .........................................................29

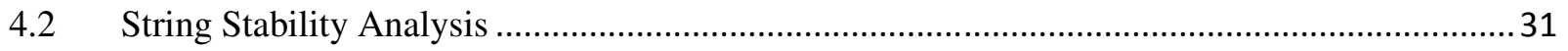

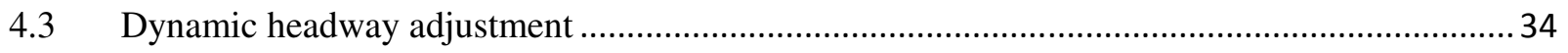

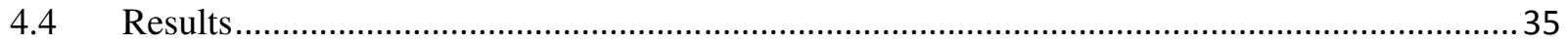




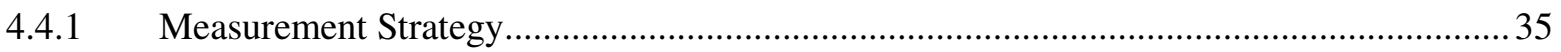

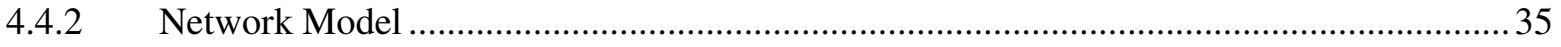

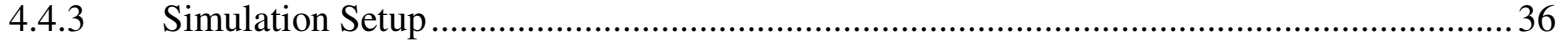

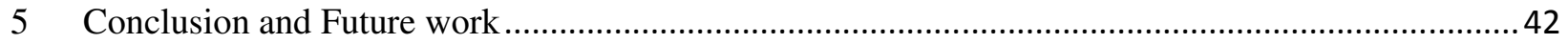

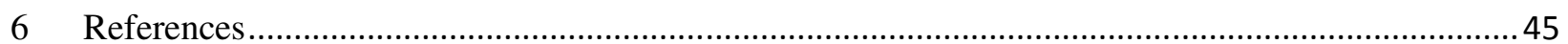




\section{List of Figures}

Fig. 1. Ns-3 Basic Model: Communication between two nodes............................................................. 13

Fig. 2. Utilization of multi-hop vehicular information for safety applications ........................................ 14

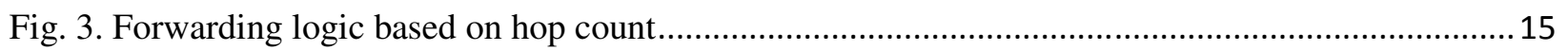

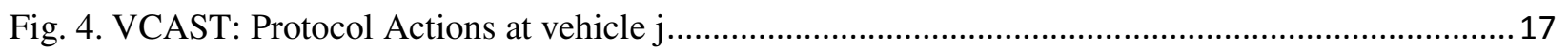

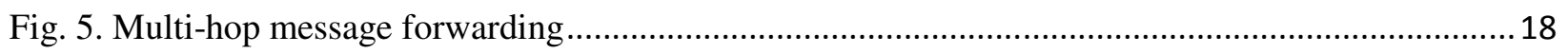

Fig. 6. Constructing Forwarding list in Timer Fired handler ............................................................... 18

Fig. 7. Neighborhood list update in Recv handler ......................................................................... 19

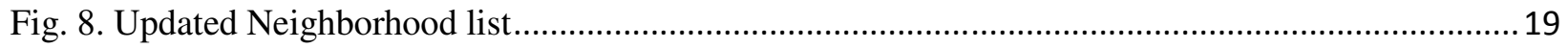

Fig. 9. Impact of network size: VCAST vs non distance-sensitive forwarding ..........................................2 21

Fig. 10. Maximum staleness vs pair-wise vehicular distance for VCAST ..............................................2 22

Fig. 11. Impact of source broadcast rate: VCAST vs non distance-sensitive forwarding ..........................2 23

Fig. 12. Comparison of message complexity: VCAST vs non distance-sensitive forwarding ...................23

Fig. 13. Impact of time varying random mobility pattern on VCAST ....................................................24

Fig. 14. Impact of time varying random mobility pattern on VCAST .....................................................25

Fig. 15. Response of followers when lead vehicle 0 detects an incident ...............................................26

Fig. 16. Timing relation of Update time $\Delta t$, Reaction time $R$ and First order time constant $\tau$.................28

Fig. 17. Indexing scheme used in car following models................................................................... 28

Fig. 18. The upper bound on $R$ as a function of $\tau$ for different acceleration feedback control $\xi \ldots \ldots . . . . . . . . .32$

Fig. 19. Rate error of vehicle 2 is the velocity difference between vehicle 2 and vehicle 1 ..................... 33

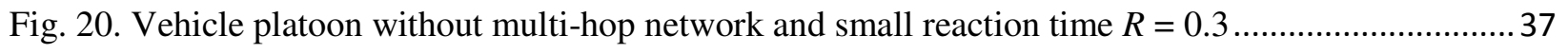

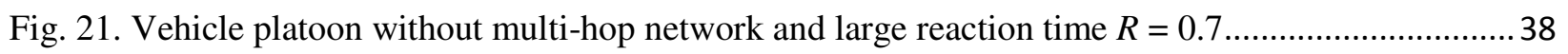

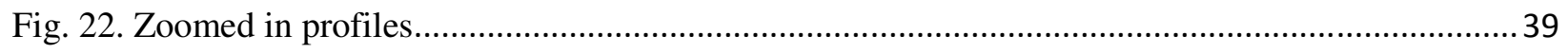

Fig. 23. Vehicle platoon in multi-hop network when incident detected. Reaction time $R=0.7 \ldots \ldots \ldots \ldots \ldots . . .40$

Fig. 24. All but first five vehicles are in multi-hop network when incident detected ............................... 41

Fig. 25. Vehicle platoon in multi-hop network when resuming normal traffic ....................................... 42 


\section{List of Tables}

Table 1. Simulation parameters for the chosen car following model..................................................... 31 


\section{Introduction}

\subsection{Problem Statement}

Vehicle Safety Application (VSA) is a well researched sub-area of Intelligent Transportation System (ITS). The core of ITS is the application of information and control technologies to transport systems to make it safe, efficient, reliable and autonomous. Many transportation problems arise from the lack of timely and accurate information, or from the lack of appropriate coordination among drivers, traffic coordinators and infrastructure. ITS is a growing field and relies on a wide range of enabling technologies to solve transportation problems. One such technology that is getting more focus recently is the infrastructure-less vehicle to vehicle $(\mathrm{V} 2 \mathrm{~V})$ wireless communication. $\mathrm{V} 2 \mathrm{~V}$ communication has countless applications and Vehicle Safety is the most critical of them all. Forward Collision Warning (FCW), Intersection Warning, Lane Change Warning, Lane Merge Warning and Stopped Vehicle Alerts are all examples of VSA. It is well known that some factors affect driver's ability to drive safely. Factors such as bad weather, poor visibility, poor road conditions, chaotic traffic and momentary distraction are major causes of road accidents. VSAs allow drivers to take more informed decision based on the knowledge of traffic movement and surrounding environment. Hence, VSA is extremely important to improve road safety and save lives. The focus of our thesis is mainly for FCW systems. But the idea can be applied to any type of VSAs.

Vehicle safety has been addressed in many ways. One of the most popular technologies that have been adopted commercially is the Adaptive Cruise Controller (ACC). ACC has a limited sensing field and in most cases look only at the predecessor vehicle. The problem with this approach is that the external delays i.e. sensing + actuation delays get accumulated in a staggered fashion beyond the sensing field which ultimately leads to rear end collision. This approach works only if there is sufficient gap between vehicles or if the external delays are sufficiently small. But factors such as low visibility, winding roads, substandard equipments, mechanical failures etc make it impossible to guarantee such conditions. Hence 
there remains a need for some form of vehicle communication which can communicate with vehicles far beyond its sensing field.

The most common form of vehicle communication is single hop broadcast of safety messages. Single hop broadcasting has a problem. All vehicles inside single hop range are safe; vehicles outside this range are unsafe as they all have to rely on predecessor look-ahead approach similar to ACC vehicles described above. Existing techniques have primarily focused on balancing transmission rate and range of single-hop broadcast. In reality, there exists an upper bound on the broadcast range for a given broadcast rate beyond which the communication degrades due to wireless channel contention. Increasing the range will cause more channel contention and reduce the information supply rate from each vehicle. Decreasing the range will also reduce the information supply rate from distant vehicles which are out of range. In this respect, single hop broadcasting seems incapable of ensuring safety of large vehicle platoons. Hence there is a need for multi hop vehicular network in vehicle communication.

Designing wireless multi-hop vehicle network for safety is challenging because it has to satisfy two critical constraints (1) acquire real-time information from immediate neighbors to react quickly to emergencies (2) acquire timely information from vehicles at large distances to plan actions in advance. The technique should be scalable for large vehicle platoons. Information rate priority tells us that the technique should also be distance sensitive which gives higher priority to closer neighbors than farther ones. There exist extensive studies of multi hop broadcasting techniques for Mobile Ad Hoc Network (MANET). However a comprehensive study of multi hop broadcasting technique that satisfies vehicle safety constraints is lacking. Hence, there remains a need to design multi-hop broadcasting techniques that are able to supply timely information over large distances without compromising on data supply rates at smaller distances.

In recent times, Vehicle Safety Applications have been investigated using either (1) Network Simulator with offline vehicle traces or (2) Traffic simulator with no network service. In real world, it is important 
to note that network service and traffic simulation impact one another and cannot be separated. The driving behavior depends upon the message propagation, whether it's a safety message or a navigational message. The message propagation in-turn depends upon vehicle motion and density in a vehicular network. Hence there is a need to study vehicle safety where both network service and traffic simulator impact one another.

In this thesis we plan to analyze the impact of wireless multi-hop network on vehicle safety through two separate but related studies. First we analyze the impact of distance sensitive multi-hop V2V network VCAST on realistic 2D traffic network. This is to measure the effectiveness of VCAST in Vehicle Safety Applications. Second we analyze the impact of multi-hop network on vehicle safety. The focus here is to define a metric to measure vehicle safety in the presence of multi-hop network. The analysis shows that multi-hop communication can prevent rear end collisions and stop and go traffic formation. The result of our study holds true for automated driving platoons as well as manual driving with Collision Warning Systems.

\subsection{Contributions}

We conducted our analysis in two parts. First we analyzed the performance and scalability of VCAST, a multi-hop distance sensitive scalable forwarding protocol. VCAST propagates information about each vehicle at a rate that decreases linearly with the distance from the vehicle. In this thesis, the performance and scalability of VCAST is validated using realistic $2 \mathrm{~d}$ traffic model which simulates vehicle movements in real traffic. The simulations are performed in ns-3, a discrete-event network simulator for Internet systems, under different network sizes, network densities, source broadcast rates and communication ranges. The results of our evaluation show that distance-sensitive forwarding is able to scale to larger network sizes as well as support much higher broadcast rates compared to non distancesensitive techniques. The reason for scalability was shown to be the significantly lower message overhead which reduces the wireless channel contention in the network. We have also characterized the performance under severe random mobility. 
In the second part, we analyzed the impact of VCAST on vehicular safety. To measure vehicle safety, we used string stability analysis. In a string stable platoon of vehicles, any perturbation in the string attenuates upstream and eventually fades away. A string stable platoon guarantees safety from rear-end collisions. Danger from rear-end collision appears only when vehicles travel close to each other. As the goal of ITS is to ultimately design autonomous driving systems which enables vehicles to run close to one another in order to increase traffic throughput and average travel time, close running vehicles will be reality in near future. We used simulations to test vehicle safety in multi-hop distance sensitive network. First, we implemented a realistic car following model which has all the essential parameters for controlling vehicle dynamics and driving behavior. Then we performed string stability analysis on a platoon of vehicle using this model. Finally, through extensive simulations we showed how string instabilities can be removed using multi-hop network service.

\subsection{Motivation}

The National Highway Traffic Safety Administration estimates that more than 90 percent of road crashes involve human error [46]. According to American Automobile Association, almost 33,000 people die on the roads in the United States each year, at a cost of $\$ 300$ billion[46]. The World Health Organization estimates that worldwide over 1.2 million people die on roads every year. VSS are already showing their impact on road safety. Data published last year by the Insurance Institute for Highway Safety [46], a U.S. nonprofit funded by the auto industry, shows that cars with forward collision warning systems, which either warn the driver about an impending crash or apply the brakes automatically, are involved in far fewer crashes than cars without them.

Similarly, VSA has other benefits. Experiments have shown that vehicles traveling in high-speed automated "platoons," reduce aerodynamic drag and could lower fuel consumption by 20 percent. And an engineering study published last year concluded that automation could theoretically allow nearly four times as many cars to travel on a given stretch of highway. That could save 5.5 billion hours of travel time 
and 2.9 billion gallons of fuel that are wasted by traffic congestion each year. If VSS is implemented and if it becomes ubiquitous, the benefits to society will be huge.

\subsection{Organization Of Thesis}

The thesis is organized as follows. Section 2 contains related work. Section 3 contains technology review. In section 4 , impact of distance sensitive multi-hop network is investigated on $2 \mathrm{D}$ traffic network. In section 5, vehicle safety is investigated in the presence of multi-hop network using string stability as a metric. Section 5 contains conclusion and future works.

\section{Related Work}

In this section we first discuss the related work in vehicle communication and vehicle safety analysis followed by related technology background. We start the section with an overview of Intelligent Transportation System (ITS) which uses vehicle communication technologies. We will then discuss in detail the existing protocols for message routing in vehicular network. This is followed by a brief introduction of network simulator Ns3 and micro traffic simulation. In the end we discuss related work.

\subsection{Distance Sensitive Wireless Multi-Hop Network For VANET}

There exists several routing protocols for vehicular ad-hoc network and a good survey of these techniques can be found in [7]-[9]. Many of these protocols have focused on delivering aperiodic, low bandwidth data to either a single vehicle (unicast) or a group of vehicles in a geographic region (geocast) with low latency [10]-[17]. In this thesis, we are primarily interested in routing techniques which support broadcast from each vehicle at high rates fit for designing VSS. Similarly, there are several protocols for single-hop V2V wireless broadcast [1], [18]-[22]. There have been several recent papers that have focused on the problem of balancing broadcast range and reliability so as to maximize the number of successful receptions in close proximity of the sender [18]-[21].A common technique used in most of these researches is to reduce the communication range in regions of high density to improve the reliability 
of reception. The trade-off in single-hop broadcast scheme is that a higher communication range reduces information availability even for nearby vehicles, while a lower communication range prevents information availability from beyond that range. The network service studied in this thesis can be used to propagate individual vehicle information over several communications hops and yet retain high data supply rates at smaller distances. There are many multi-hop broadcast algorithms proposed for MANETs and Wireless Sensor Networks (WSN) as listed in [23]-[29]. Most of these algorithms have been extended for vehicular network with mobile nodes. Most of them achieve multi-hop forwarding by choosing optimal forwarding node and proposing heuristics for reducing redundant forwarding. A good survey of multi-hop broadcast techniques in mobile ad-hoc networks is presented in [31]. The concept of distance-sensitivity has found applications before in several other networking fields in different forms- like propagating routing table [32], [33], querying and tracking applications [35]-[38], supplying global state information to all nodes [39] etc.

A unique feature of VCAST that existing algorithms do not talk about is - data forwarding with adaptive broadcasting rate that depends on distance. This feature is important for scalability and reliable VSS implementation. There exists an extensive study of VCAST performance in 1d single road segment with multiple lanes [3]. The performance study in realistic $2 \mathrm{~d}$ traffic network has not been explored before, which is the focus of this thesis.

\subsection{Multi-Hop Network In Vehicular Safety Analysis}

There have been many studies of string stability for Adaptive Cruise Controlled (ACC) vehicles. [41] shows that to achieve string stability with constant inter-vehicle spacing, vehicle-to-vehicle communication is necessary. [42] uses a simple spring-mass-damper system to demonstrate the idea of string stability and show the string-stability criterion for constant time headway and variable timeheadway policies. [43] proved, among many other interesting things that if the coupling between two vehicles is weak enough, the controlled system is string stable. [44] gives the upper bound on reaction 
time and first order time constant for a string of ACC vehicles. The proof is shown analytically as well as through simulations. All the above techniques use vehicles which look at only the predecessor's state for making control decisions. There is no communication used and predecessor's states are read by follower's sensors, like RADAR, LIDAR or video camera. The drivers of ACC equipped vehicles do not have advance information of events occurring far ahead or beyond their sensory field. This is true especially in bad weather conditions, winding roads, urban traffic where the sensors accuracy could get reduced. As a result, drivers get less time to react. And it could result in rear-end collisions.

Interestingly, some studies have utilized single-hop broadcast network service in the design of Safety Systems [45]. Problem with single-hop broadcast is that it needs higher broadcast range for large platoon size. There exists an upper bound on the broadcast range for a given broadcast rate beyond which the communication degrades due to wireless channel contention. Such approach is not scalable to real world situation where platoon size varies arbitrarily. Also, some areas have many obstructions- like winding hilly roads and cities with tall buildings. In such areas, single-hop broadcast is not a reliable solution. Hence there is a need for studying the impact of Vehicle Safety System that utilizes multi-hop wireless network.

\subsection{Intelligent Transportation Systems}

Intelligent Transport Systems (ITS) is a broad term used for the integrated application of communications, control and information processing technologies to the transportation system. ITS covers all modes of transport and considers all elements of the transportation system - the vehicle, the infrastructure, and the driver, interacting together dynamically. Many ITS tools are based on the collection, processing, integration and supply of information. Data generated by ITS provide real-time information about current conditions of the traffic, or on-line information for journey planning. Goal of ITS is to make transportation safe, efficient and also to save energy and environment.

ITS uses a wide range of technologies and functions. Some of these technologies are listed below. 


\section{- Communications}

Various forms of wireless communication technologies have been proposed. Some of the popular ones are WAVE/DSRC for vehicle to vehicle communication and WiMAX, GSM, 3G for realtime travel information; fleet management; emergency response.

\section{- Processing Technologies}

Various processing technologies include microprocessor with hardware memory management, Real-time Operating Systems and softwares with Artificial Intelligence.

\section{- Geographical Location}

This technology is used for obtaining travel time and speed data for vehicles traveling along streets, highways, freeways, and other transportation routes. Various methods used are triangulation methods, GPS based methods, RFID etc. This technology is used for tracking and tracing vehicles.

\section{- Sensing Technologies}

Various sensing technologies used are RADAR, LIDAR, video cameras, and inductive loops. Some are used for vehicle safety and some are used for vehicle detection.

\section{- Actuation Technologies}

Most popular actuation technologies used are Automatic Braking System (ABS) and Adaptive Cruise Control (ACC). An important characteristic of many actuation technologies is their potential for two-stage operation: initially advisory and then, if the driver fails to respond, interventionist.

ITS applications can be categorized under several classes. These applications are not necessarily mutually exclusive, but work synergistically to achieve ITS objectives.

\section{- Traffic Congestion Monitoring}


Various applications include highway incident detection and management, 'green waves' traffic signaling to give priority to emergency vehicles, traffic signal coordination to minimize traffic delays and queues, rerouting guidance etc.

\section{- Active Safety}

Various applications include Emergency Brake Light Warning, Forward Collision Warning, Intersection Movement Assist, Blind Spot and Lane Change Warning, Driver drowsiness detectors etc.

\section{- Environmental Monitoring}

Various applications include Pollution detection, Electronic pollution charging for high pollution areas etc.

\section{- Information Services}

Various applications include Multimedia, Navigation applications, Real-time traffic update applications etc.

\section{- Administrative Services}

Various applications include Electronic payment, Automatic road enforcement, Variable speed limits etc.

\subsection{Vehicle Communication}

There are typically two types of vehicle communication - Vehicle to Infrastructure (V2I) communication and vehicle to vehicle $(\mathrm{V} 2 \mathrm{~V})$ communication. V2I communication has two types of infrastructure - Road Side Equipments (RSU) and Base Stations (BS). RSUs include traffic signals, toll gates, road sensors etc. Communication between vehicle and RSU uses Dedicated Short Range Communication (DSRC). DSRC is a low bandwidth, low range wireless communication. Communication between vehicle and BS uses mobile communication technologies. Communication between RSU and BS uses wired fiber optic or mobile communication such as 3G, 4G, WiMAX and LTE. V2V communication only uses DSRC. 
The standard used for vehicle communication is IEEE $802.11 \mathrm{p}$ also popularly called Wireless Access in Vehicular Environments (WAVE). It defines enhancements to 802.11 required for data exchange between high-speed vehicles and between the vehicles and the roadside infrastructure in the licensed ITS band of $5.9 \mathrm{GHz}(5.85-5.925 \mathrm{GHz})$. IEEE 1609 is a higher layer standard based on the IEEE 802.11p. The licensed band is for DSRC devices.

\subsection{Vehicle Network And Routing}

Vehicular Area Network (VANET) is a special case of Mobile Ad-hoc NETwork (MANET). Mobile nodes of MANET are replaced by cars in VANET. Like MANETs, VANETs are distributed, selforganizing communication networks. VANETs posses few distinguishing characteristics that makes them challenging than MANETs. (1) The nodes have dynamic topology because of the vehicles speed. (2) Dynamic topology results in frequently disconnected network. A robust VANET routing protocol needs to recognize the frequent disconnectivity and provide an alternative link quickly to ensure uninterrupted communication.(3) Vehicles follow a certain mobility pattern that is a function of the underlying roads, the traffic lights, the speed limit, traffic condition, and drivers' driving behaviors. VANET routing should be tested under realistic mobility traces. (4) Nodes in VANET have unlimited battery power and storage. Therefore existing optimal duty cycle techniques are not relevant in VANET. (5) Unlike MANETs, nodes in VANETs assume to have on-board GPS units which can be utilized in routing decisions.

A routing protocol defines the procedure to establish a route, make forwarding decision, maintain the route once established and recover from routing failures. VANET routing protocols can be broadly classified into two types - Topology based and Position based (Geographic) routing. Topology based routing can be further divided into Proactive (table-driven) and Reactive (on-demand) routing. Proactive routing maintains a table in the background by constantly broadcasting control packets. Advantage is low latency. Drawback is maintenance of routing path for non-used paths, which could waste a lot of resource for highly dynamic topology in VANET. Fisheye State Routing (FSR) is an example of Proactive routing. FSR is an efficient Link State Routing protocol where it broadcasts immediate neighbor's link state with 
decreasing frequency as hop distance from source increases. Reactive routing creates routing table only when it is necessary for nodes to communicate by flooding Query and Reply packets. Reactive routing has high latency but optimum resource utilization. Ad Hoc On-Demand Distance Vector routing (AODV) is one such example. It records a backward path as Query packets propagate from source to destination and a forward path as Reply packets propagate from destination to source. Dynamic Source Routing (DSR) is another example where Query packets record IDs of all the intermediate nodes and the destination constructs a routing path from the header of this Query packet. Another example Temporally Ordered Routing Algorithm (TORA) routing creates a directed acyclic graph (DAG) toward the destination based on the height of the tree rooted at the source. A node will rebroadcast a Query packet only if it has a downward link to the destination, else packet is dropped. Drawback of TORA is that it has route to all nodes, so maintenance is heavy.

Position based or Geographic routing algorithms use position information such as GPS coordinates for making packet forwarding decisions. They do not need to build routing tables or exchange link states. A common approach in all the geographic routing algorithms is to use a greedy strategy to select a next hop node e.g. selecting neighbor closest to the destination. However, greedy forwarding fails in local minimum situations, e.g. when reaching a node that is closer to the destination than all its neighbors. There should be a recovery strategy for this situation. One such widely adopted strategy is Face routing. Greedy Perimeter Stateless Routing (GPSR) is an example. The above strategy requires nodes to know the locations of its neighbors and hence use beacons to exchange the location information. Beacon-less Geographic routing is also possible where next hop choice is contention based. They set a timer in accordance to their distance to the destination. Once the first timer expires, the respective candidate retransmits the message. The other candidates overhear the re-transmission and cancel their scheduled transmissions. This strategy causes transmission redundancy if two nodes transmit near simultaneously. This could be solved by using hop counter in each packet. A candidate cancels the scheduled transmission if a node retransmitted the packet with same hop count. Beacon-less routing should also have a recovery 
strategy in-order to solve local minimum situations. Contention-based Forwarding (CBF) and Implicit Geographic Forwarding (IGF) are all examples of beacon-less geographic routing.

\subsection{Network Simulation And Ns3}

A network simulator is a software program that imitates the working of a computer network. The computer network is modeled by using mathematical formula or actually capturing and playing back observations from a production network. Network simulators save time and cost of setting up a test bed containing multiple computers, routers and data links. Some test scenarios are not only expensive but difficult to emulate using real hardware - for instance simulating a scenario with several thousand nodes or experimenting with a new protocol in the network. Network simulators are useful in such situations. A network simulator in minimum must enable users to create network nodes, add links between these nodes, put the nodes in some network topology and generate data traffic between the nodes.

Ns-3 is a discrete-event network simulator. In discrete event simulation, a list of pending "events" is stored, and those events are processed in order. Each event occurs at a particular instant in time and marks a change of state in the system. Between consecutive events, no change in the system is assumed to occur; thus the simulation can directly jump in time from one event to the next. Ns-3 is written entirely in C++ with bindings for Python. Ns-3 uses waf build system. Simulation programs are C++ executables or python scripts. Fundamental components of Ns-3 include

- Node: It represents the motherboard of a computer with RAM, CPU, and IO interfaces. Each node can support multiple NetDevices.

- Application: A packet generator and consumer which can run on a Node and talk to a set of network stacks.

- NetDevice: Represents a network card which can be plugged in an IO interface of a Node. NetDevices are strongly bound to Channels of a matching type. For example WifiNetDevice can operate only in WifiChannel and CsmaNetDevice can operate only in CsmaChannel. 
- Channel: Represents a physical connector between a set of NetDevice objects

- Protocol Stack: Represents layer 3, layer 4 and routing. It implements Ipv4, TCP and UDP.

- Simulation Core: Manages events, scheduler, tracing, callbacks etc.

- Mobility Model: This component is useful for VANET simulation. It provides mobility to nodes. Example constant velocity model, random walk model, random direction model etc.

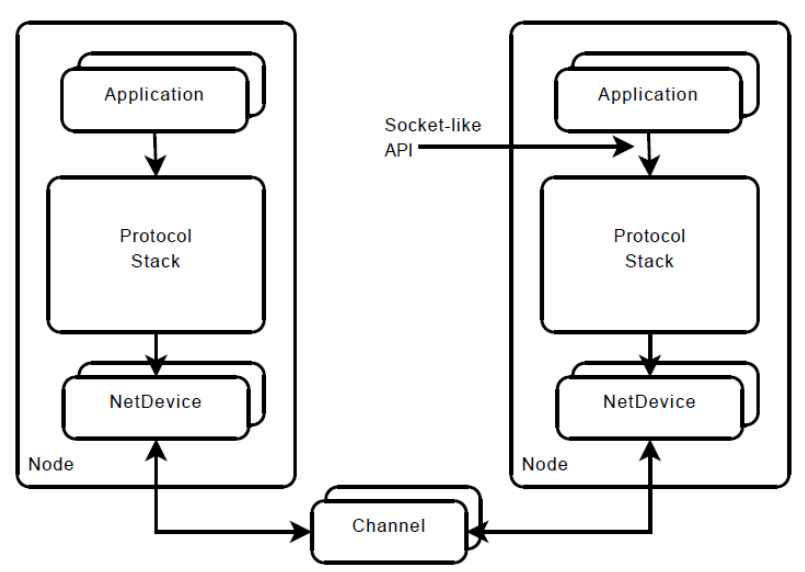

Fig. 1. Ns-3 Basic Model: Communication between two nodes

Fig. 1 shows the basic model of Ns-3 and how the fundamental components interact. Ns-3 has a layered architecture and has a rich set of libraries for each layer. Its socket interface is very similar to BSD socket. The network creation and configuration file is written just like a script and is easy to understand. Ns-3 also has extensive documentation and a large user base. Because of these factors, Ns-3 has been widely adopted as a network simulation tool both by academy and the industry.

\subsection{Micro-Traffic Simulation}

Traffic simulation is a tool for investigating traffic systems. There are two major types of traffic simulation models - Micro and Macro traffic simulation. Micro traffic simulation models are used to model the behavior of each individual vehicle and Macro traffic simulation models are used to model the behavior of whole traffic system. In this thesis, we are mainly interested in the former. A micro traffic 
simulation model consists of sub-models that describe human driver behavior. Important behavior model include; gap-acceptance, speed adaptation, lane changing, ramp merging, overtakes and car-following. Most previous research on driving behavior modeling has been focused on car-following. Car-following models are commonly classified into classes depending on the utilized logic. Such model classes include:

- Gazis-Herman-Rothery models (GHR): These models state that the following vehicle's acceleration is proportional to the speed of the follower, the speed difference between follower and leader and the space headway.

- Safety-distance models: Safety distance models are based on the assumption that the follower always keeps a safe distance to the vehicle in front.

- Psycho-physical car-following models: These models use thresholds for e.g. the minimum speed difference between follower and leader perceived by the follower.

\section{Analyzing the Performance and Scalability of VCAST, a distance sensitive multi-hop broadcast protocol}

Before proceeding further, let us give a brief description of how VCAST performs distance sensitive broadcasting. Fig. 1 shows a possible scenario where VCAST can be useful.

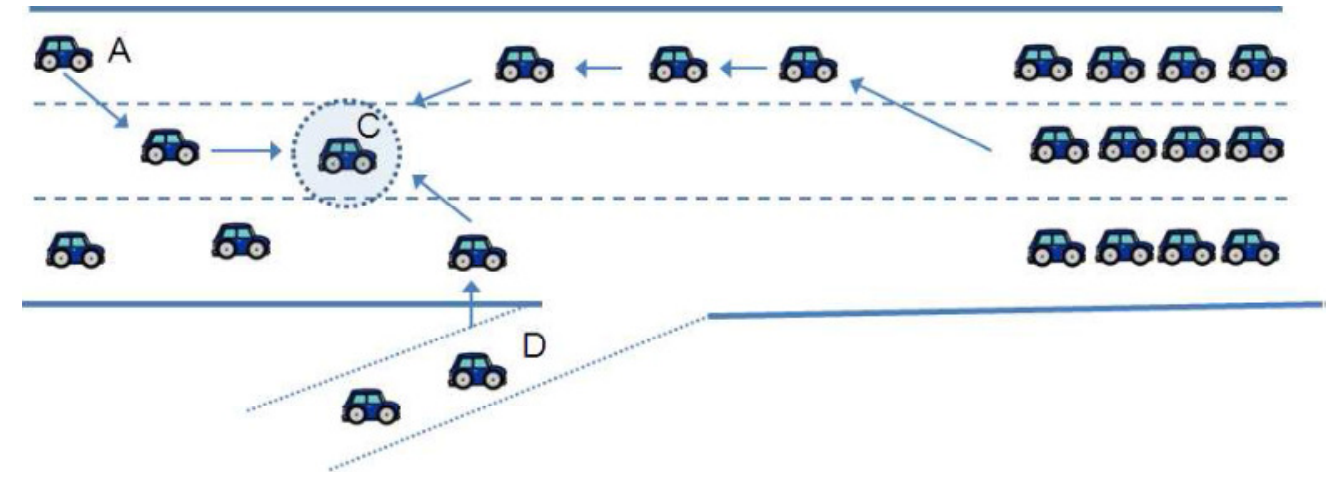

Fig. 2. Utilization of multi-hop vehicular information for safety applications: The arrows indicate multi-hop information flow towards car $C$. Knowledge of states of $A$ and $D$ will guarantee safe lane change to left and right respectively. Knowledge of congestion (shown by dense cluster of vehicles) will allow safe and timely reaction. 


\subsection{Distance Sensitive Broadcast Algorithm}

The VCAST algorithm has distance sensitive property. The rate of forwarding decreases linearly with the distance of source from the forwarding vehicle. It is achieved by forwarding the information of the vehicle at a distance of ' $h$ ' hops only once in every ' $h$ ' transmit cycles. Fig. 2 shows what hop counts are forwarded in each Transmit Cycle. The figure shows only the first ten transmit cycles. Transmit Cycle is also called Sequence Number. VCAST also suppresses forwarding of information about a vehicle in an interval, if some other vehicle has already forwarded information about the respective vehicle in that interval.

\begin{tabular}{|l|l|l|l|l|l|l|l|l|l|l|}
\hline $\begin{array}{l}\text { Transmit } \\
\text { Cycle } \\
\text { No }\end{array}$ & \multicolumn{7}{|l|}{$\begin{array}{l}\text { Hop count of nodes whose information are } \\
\text { forwarded }\end{array}$} \\
\hline 1 & 1 & & & & & & & & & \\
\hline 2 & 1 & 2 & & & & & & & & \\
\hline 3 & 1 & & 3 & & & & & & & \\
\hline 4 & 1 & 2 & & 4 & & & & & & \\
\hline 5 & 1 & & & & 5 & & & & & \\
\hline 6 & 1 & 2 & 3 & & & 6 & & & & \\
\hline 7 & 1 & & & & & & 7 & & & \\
\hline 8 & 1 & 2 & & 4 & & & & 8 & & \\
\hline 9 & 1 & & 3 & & & & & & 9 & \\
\hline 10 & 1 & 2 & & & 5 & & & & & 10 \\
\hline
\end{tabular}

Fig. 3. Forwarding logic based on hop count: Transmit Cycle No increments by 1 for every broadcast. In every transmit cycle, only those hop counts are forwarded which are factors of that transmit cycle.

Staleness in vehicular state information is used as a metric for information quality as it reflects how old the current information about a particular vehicle is. The staleness $S(j, i, t)$ in the state of vehicle $i$ as possessed by vehicle $\mathrm{j}$ at time $\mathrm{t}$ is the time elapsed since the timestamp of the state of $\mathrm{i}\left(\mathrm{j} \cdot \mathrm{T}_{\mathrm{i}}\right)$. Thus $\mathrm{S}(\mathrm{j}, \mathrm{i}, \mathrm{t})$ $=\mathrm{t}-\mathrm{j} \cdot \mathrm{T}_{\mathrm{i}}$. It is proved that traffic information in VCAST can be obtained with a worst-case staleness that is bounded by $\mathrm{O}\left(\mathrm{d}^{2}\right)$ where $\mathrm{d}$ is the distance from the source of the information. Thus, VCAST provides traffic information which is independent of traffic density and network size. At the same time, the average 
communication cost (the required transmission rate at each node) also is only bounded by $\mathrm{O}(\mathrm{p} \sqrt{N})$, where $\mathrm{p}$ is the broadcast rate at the source, $\mathrm{N}$ is total nodes. Lower communication overhead per node ensures lower channel contention and higher source broadcast rates, thus benefiting information supply at smaller distances.

VCAST algorithm is described as follows. Every node has two lists - Neighborhood list and a Forwarding list. Forwarding list contains the list of nodes whose information should be forwarded and is created inside Timer Fired event handler. Whereas Neighborhood list keeps track of all the vehicles in its tracking zone and is populated by Recv event handler. The algorithm at node 'j' starts with variable initialization. The counters and the sequence number are set to zero. The vehicle list is initialized by adding itself to the list. This list is a persistent list. Then the transmit timer is initialized with a period of $1 / \mathrm{p}$. When the transmit timer fires, the code goes into Timer fired event handler. The execution in timer fired event handler starts by increasing the sequence number. Then the node starts building the Forwarding list. First, the node adds itself to the Forwarding list as its own information should be propagated. Then for all nodes $\mathrm{i}$ in the Persistent list, only add those nodes in the Forwarding list whose hop count is equal to multiple of sequence number and whose information is not received from more than one source since j's last transmission. If node i's information is forwarded, its counter is cleared to zero. Recv event handler is triggered when the node receives packet. In the Recv event handler, the node updates entries in Neighborhood list only if the time stamps of received nodes are greater than the nodes currently in Neighborhood list. Upon successful update, counters are initialized to 1. A new node can also be added to Neighborhood list at this stage and its counter is also initialized to 1. Nodes whose time stamps are equal, their counter is incremented by 1 . This ensures the node doesn't forward that information again as it is already being circulated. Nodes whose time stamps are less than the nodes in Neighborhood list, they are simply discarded. This will ensure that in the next transmission a much newer version of information is circulated subsiding the older information. 


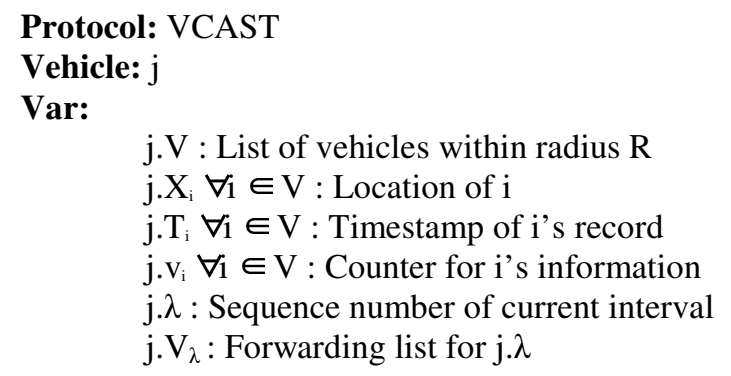

Actions:

$\left\langle\mathrm{A}_{1}\right\rangle::$ Initialization: $\rightarrow$

$j . V=j ; j . V i=0 ; j . \lambda .=0 ;$

Timer.start $\left(\frac{1}{p}\right)$

$\left\langle\mathrm{A}_{2}\right\rangle::$ Timer fired: $\rightarrow$

j. $\lambda=$ j. $\lambda+1$

$\mathrm{j} . \mathrm{V}_{\lambda}=\mathrm{j}$

$\forall \mathrm{i} \in \mathrm{j} . \mathrm{V}$

if $(\lambda \bmod d h(i, j)==0)$

if $j . v i<2$

Add $\mathrm{i}$ to $\mathrm{j} . \mathrm{V}_{\lambda}$

fi

$j \cdot v i=0$;

fi

$\forall \mathbf{i} \in \mathrm{j} . \mathrm{V}_{\cdot \lambda}$

Send j.Xi, j.Ti

$\left\langle A_{3}\right\rangle:: \operatorname{recvi}(V) \rightarrow$

$\forall \mathrm{k} \in \mathrm{i} . \mathrm{V}$

if $\left(\left(j . T_{k}<i . T_{k}\right) \vee(k ! \in j . V)\right)$

fi j. $X_{k}=i . X_{k} ; j . T_{k}=i . T_{k} ; j . V_{k}=1$;

elseif $\left(j . T_{k}==i . T_{k}\right)$

fi

$j \cdot v k=j . v k+1$

Fig. 4. VCAST: Protocol Actions at vehicle j

Let's look at an example how Timer Fired Handler builds a Forwarding List in an arbitrary node. Let us assume that the node is in $8^{\text {th }}$ transmit cycle. At this cycle, only thee nodes with hop counts $1,2,4$ and 8 are to be forwarded. Let the populated Neighborhood List have following entries as shown in Fig. 4(b). 


\begin{tabular}{|l|l|l|l|l|l|l|l|l|l|l|}
\hline $\begin{array}{l}\text { Transmit } \\
\text { Cycle } \\
\text { No }\end{array}$ & \multicolumn{6}{|l|}{$\begin{array}{l}\text { Hop count of nodes whose information are } \\
\text { forwarded }\end{array}$} \\
\hline 1 & 1 & & & & & & & & & \\
\hline 2 & 1 & 2 & & & & & & & & \\
\hline 3 & 1 & & 3 & & & & & & & \\
\hline 4 & 1 & 2 & & 4 & & & & & & \\
\hline 5 & 1 & & & & 5 & & & & & \\
\hline 6 & 1 & 2 & 3 & & & 6 & & & & \\
\hline 7 & 1 & & & & & & 7 & & & \\
\hline 8 & 1 & 2 & & 4 & & & & 8 & & \\
\hline 9 & 1 & & 3 & & & & & & 9 & \\
\hline 10 & 1 & 2 & & & 5 & & & & & 10 \\
\hline
\end{tabular}

(a)

\begin{tabular}{|c|c|c|c|}
\hline $\begin{array}{c}\text { Hop } \\
1\end{array}$ & $\begin{array}{c}\text { Hop } \\
2\end{array}$ & $\begin{array}{c}\text { Hop } \\
4\end{array}$ & $\begin{array}{c}\text { Hop } \\
5\end{array}$ \\
\hline $\mathrm{T}=3$ & $\mathrm{~T}=2$ & $\mathrm{~T}=2$ & $\mathrm{~T}=3$ \\
\hline $\mathrm{v}=1$ & $\mathrm{v}=2$ & $\mathrm{v}=1$ & $\mathrm{v}=1$ \\
\hline
\end{tabular}

(b)

Fig. 5. Multi-hop message forwarding (a) Forwarding logic based on transmit cycle and hop count (b) Neighborhood List: List is currently populated with entries of self and nodes with hop counts 1, 2, 4 and 5 .

Only nodes with hop counts 1 and 4 are added to forwarding list. Node with hop count 2 is dropped because count $>1$ and 5 is dropped because it is not it's the transmit cycle. Fig. 5 is the resultant Forwarding List. Once the nodes are chosen for forwarding, their respective counts are reset in the Neighborhood List as shown in Fig. 6.

\begin{tabular}{|c|c|}
\hline $\begin{array}{c}\text { Hop } \\
1\end{array}$ & $\begin{array}{c}\text { Hop } \\
4\end{array}$ \\
\hline $\mathrm{T}=3$ & $\mathrm{~T}=2$ \\
\hline
\end{tabular}

(a)

\begin{tabular}{|c|c|c|c|}
\hline $\begin{array}{c}\text { Hop } \\
1\end{array}$ & $\begin{array}{c}\text { Hop } \\
2\end{array}$ & $\begin{array}{c}\text { Hop } \\
4\end{array}$ & $\begin{array}{c}\text { Hop } \\
5\end{array}$ \\
\hline $\mathrm{T}=3$ & $\mathrm{~T}=2$ & $\mathrm{~T}=2$ & $\mathrm{~T}=3$ \\
\hline $\mathrm{v}=0$ & $\mathrm{v}=0$ & $\mathrm{v}=0$ & $\mathrm{v}=1$ \\
\hline
\end{tabular}

(b)

Fig. 6. Constructing Forwarding list in Timer Fired handler (a) Forwarding list (b) Updated Neighborhood list

Let's look at an example how Recv Handler updates Neighborhood list in an arbitrary node. Let us assume that Neighborhood List contains entries of self and nodes with hop count 1,2 and 4. Again, lets us assume that it receives broadcast from nodes with hop count 1,2,4 and 5 . 


\begin{tabular}{|c|c|c|}
\hline $\begin{array}{c}\text { Hop } \\
1\end{array}$ & $\begin{array}{c}\text { Hop } \\
2\end{array}$ & $\begin{array}{c}\text { Hop } \\
4\end{array}$ \\
\hline $\mathrm{T}=2$ & $\mathrm{~T}=2$ & $\mathrm{~T}=2$ \\
\hline $\mathrm{v}=1$ & $\mathrm{v}=1$ & $\mathrm{v}=1$ \\
\hline
\end{tabular}

(a)

\begin{tabular}{|c|c|c|c|}
\hline $\begin{array}{c}\text { Hop } \\
1\end{array}$ & $\begin{array}{c}\text { Hop } \\
2\end{array}$ & $\begin{array}{c}\text { Hop } \\
4\end{array}$ & $\begin{array}{c}\text { Hop } \\
5\end{array}$ \\
\hline $\mathrm{T}=3$ & $\mathrm{~T}=2$ & $\mathrm{~T}=1$ & $\mathrm{~T}=3$ \\
\hline
\end{tabular}

(b)

Fig. 7. Neighborhood list update in Recv handler (a) Neighborhood list (b) Received list

Upon comparison of Neighborhood list with received list, the following changes take place. Node with hop count 1 is a fresh broadcast; its counter is reset. Node with hop count 2 is a duplicate; its counter is incremented by 1 . Node with hop count 4 is not updated; it is an old broadcast. Node with hop count 5 is also a fresh broadcast; it is added to the list and counter is reset. Result Neighborhood list is shown in Fig. 8.

\begin{tabular}{|c|c|c|c|}
\hline $\begin{array}{l}\text { Hop } \\
1\end{array}$ & $\begin{array}{l}\text { Hop } \\
2\end{array}$ & $\begin{array}{l}\text { Hop } \\
4\end{array}$ & $\begin{array}{l}\text { Hop } \\
5\end{array}$ \\
\hline $\mathrm{T}=3$ & $\mathrm{~T}=2$ & $\mathrm{~T}=2$ & $\mathrm{~T}=3$ \\
\hline $\mathrm{V}=1$ & $\mathrm{~V}=2$ & $\mathrm{~V}=1$ & $\mathrm{v}=1$ \\
\hline
\end{tabular}

Fig. 8. Updated Neighborhood list

\subsection{Results}

VCAST is evaluated by using extensive simulations in ns-3, a discrete event simulator for wireless and mobile ad-hoc networks.

\subsubsection{Network Models}

We use an IEEE 802.11b physical layer communication model with a DSSS rate of $11 \mathrm{Mbps}$ at each node. We first consider $2 \mathrm{~d}$ traffic model with uniform mobility. For that we take vehicular traffic to be in $2 \mathrm{~d}$ grids of different sizes $(28 \times 28,25 \times 25,20 \times 20$ and $15 \times 15)$ with uniform separation between the vehicles at all times (i.e., traveling with uniform speed). We consider inter-vehicular separations of $60 \mathrm{~m}, 50 \mathrm{~m}, 40 \mathrm{~m}$ and $30 \mathrm{~m}$, thus simulating different densities. We also consider source broadcast rates of $1 \mathrm{~Hz}, 5 \mathrm{~Hz}$ and 
$10 \mathrm{~Hz}$, and communication ranges of $75 \mathrm{~m}, 100 \mathrm{~m}, 125 \mathrm{~m}$ and $150 \mathrm{~m}$, which are within the range of expected transmission rate and range values of Here I am messages for intelligent transportation systems. Next, we consider 2-d traffic model with non uniform mobility which generalizes possible vehicular traffic patterns. For this we use random way-point mobility model. The goal here is to study if time varying densities impact the performance. We use these models to compare VCAST with non distance-sensitive approaches and to systematically study the impact of density, source broadcast rates and communication range on the performance.

\subsubsection{Measurement Strategy}

Each vehicle's state information is assumed to be 10 bytes long. In any given slot, a vehicle may transmit a variable number of such records. At each vehicle, we measure the maximum staleness with respect to every other vehicle by measuring the time elapsed since the information originated at the source, just before fresh information about a vehicle is received. All simulations are run for 20 seconds except simulations at $1 \mathrm{~Hz}$ which are run for 40 second due to higher staleness values. We then group the maximum staleness based on pair wise distances between vehicle-vehicle. The average of these measurements over multiple experiments is used in our evaluations.

\subsubsection{Impact of distance sensitivity in uniform mobility}

The objective of this section is to quantify the performance gains achieved by using distancesensitive forwarding VCAST as opposed to non distance sensitive forwarding i.e. forwarding information about all vehicles at the rate equal to source broadcast rate.

\section{Scaling in number of nodes:}


Fig 9(a) shows the maximum staleness as a function of distance at $\mathrm{p}=10 \mathrm{~Hz}$ and network size of 225 nodes for both VCAST as well as non distance-sensitive forwarding. Non distance sensitive forwarding keeps staleness low at small distances and then grows linearly with distance. On the other hand, VCAST keeps staleness low at small distances but then grows quadratically i.e. $\mathrm{O}\left(\mathrm{d}^{2}\right)$ with distance, as expected. Fig 9(b) compares staleness in a larger network size of 600 nodes. Non-distance sensitive forwarding shows much higher staleness even at smaller distances and shows more than linear growth rate with distance. On the other hand, VCAST keeps staleness low at small distances and grows quadrically i.e. $\mathrm{O}\left(\mathrm{d}^{2}\right)$ with distance. Information within $400 \mathrm{~m}$ is obtained at lower than $300 \mathrm{~ms}$ using VCAST while it takes about 3 seconds in the case of a non distance-sensitive approach. This is due to the fact that as the number of nodes increases, the channel contention increases at a much higher rate in the non distancesensitive forwarding causing message losses and consequently increase in staleness. By reducing channel contention, VCAST is able to achieve scalability.

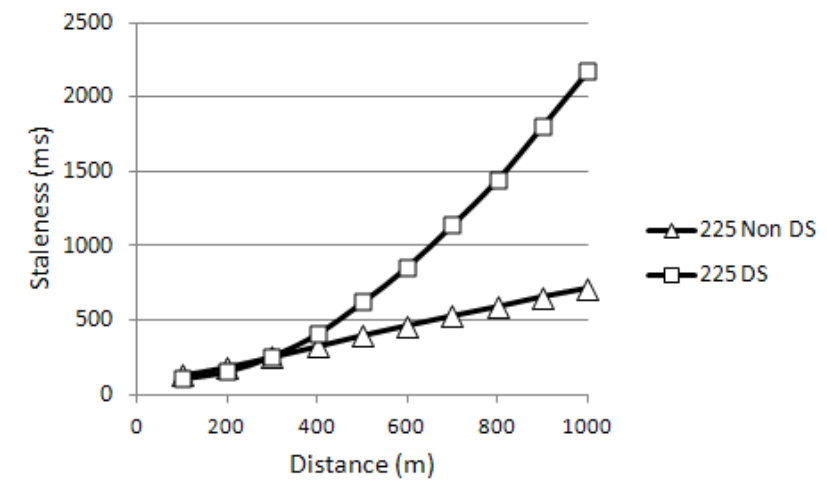

(a)

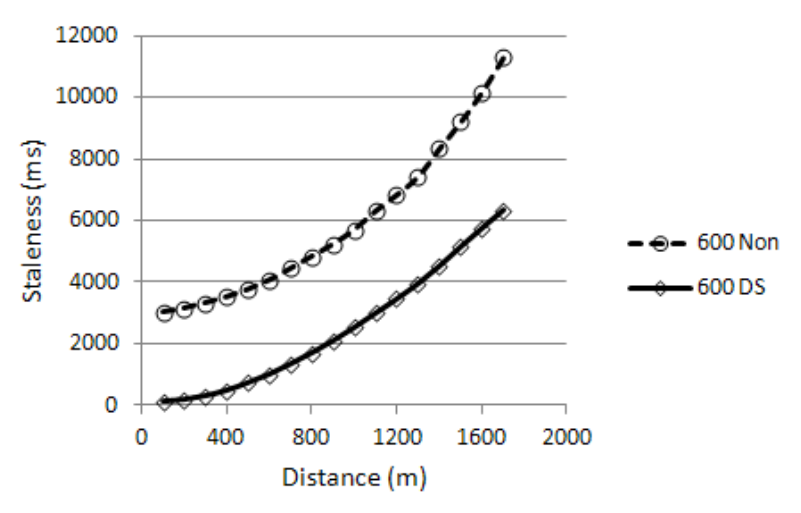

(b)

Fig. 9. Impact of network size: VCAST vs non distance-sensitive forwarding. (a) Maximum staleness vs pairwise vehicular distance: $p=10 \mathrm{~Hz}$ at 225 nodes (b) Maximum staleness vs pair-wise vehicular distance: $p=$ $10 \mathrm{~Hz}$ at 600 nodes

Fig. 10 shows the maximum staleness as a function of distance at $\mathrm{p}=10 \mathrm{~Hz}$ and network size of 225,400 , 625 and 784 nodes for VCAST. We observe that staleness values are preserved at corresponding distances, irrespective of network size. 


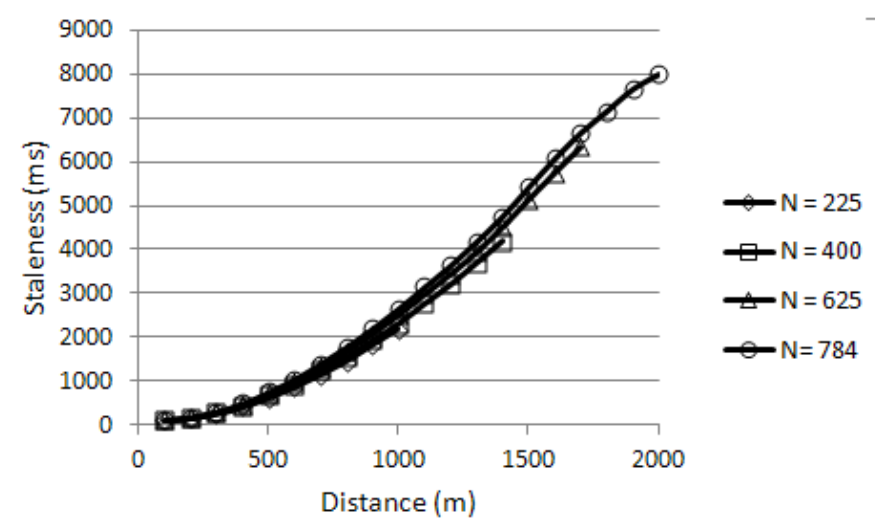

Fig. 10. Maximum staleness vs pair-wise vehicular distance for VCAST: $p=10 \mathrm{~Hz}$, network sizes of 225, 400, 625 and 784 vehicles, Communication range $100 \mathrm{~m}$, separation 50m: staleness values are preserved at corresponding inter-vehicular distances, irrespective of network size.

\section{Scaling in source broadcast rate:}

In Fig. 11(a) and Fig. 11(b), we fix the network size to 784 nodes and vary the source broadcast rate. In Fig. 11(a), we set source broadcast rate of $1 \mathrm{~Hz}$. The non distance-sensitive scheme is able to scale to large distances and has lower latencies at larger distances compared with VCAST since there is no staggered forwarding. However, when attempting to reduce the staleness at smaller distances, by increasing the source broadcast rate, non distance-sensitive forwarding fails. This is shown in Fig. 11(b) for broadcast rates of $10 \mathrm{~Hz}$. The non distance-sensitive approaches show much higher staleness even at smaller distances. On the other hand, VCAST is able to deliver lower staleness at smaller distances and progressively increasing the staleness at larger distances.

We observe that staleness is kept low even at higher source broadcast rate. This allows the network bandwidth to be utilized where it is needed and achieve scalability. 


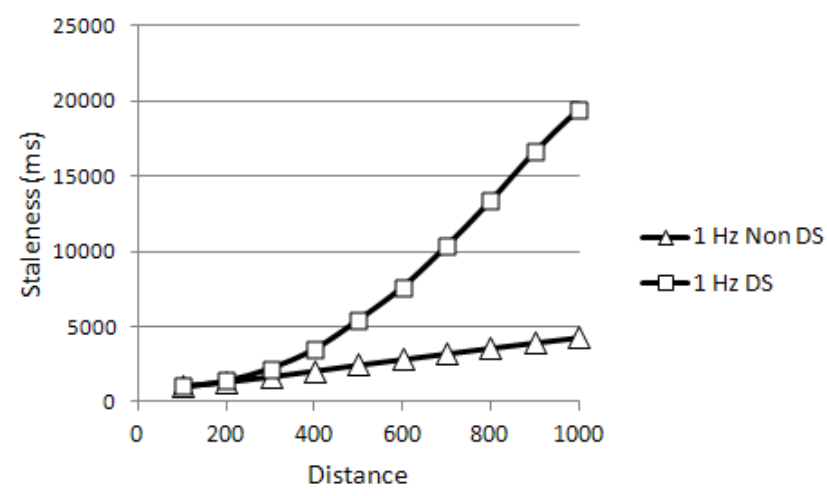

(a)

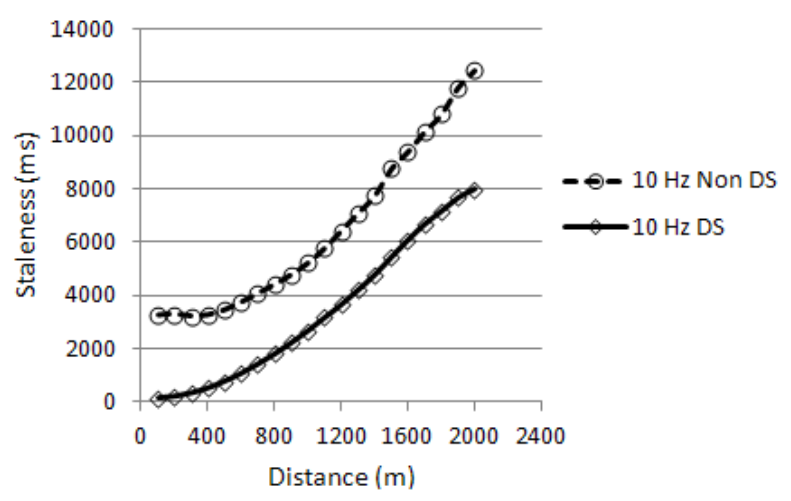

(b)

Fig. 11. Impact of source broadcast rate: VCAST vs non distance-sensitive forwarding. (a) Maximum staleness vs pair-wise vehicular distance: $p=1 \mathrm{~Hz}$ at 784 nodes (b) Maximum staleness vs pair-wise vehicular distance: $p=10 \mathrm{~Hz}$ at 784 nodes

\section{Message Complexity:}

In Fig. 12(a) and Fig. 12(b), we show the number of vehicular records transmitted per second by every node for varying network sizes and varying source broadcast rates respectively. These figures highlight the significantly lower message complexity in VCAST which reduces the contention even as network size and broadcast rates grow. This is also the reason behind successful scaling in VCAST.

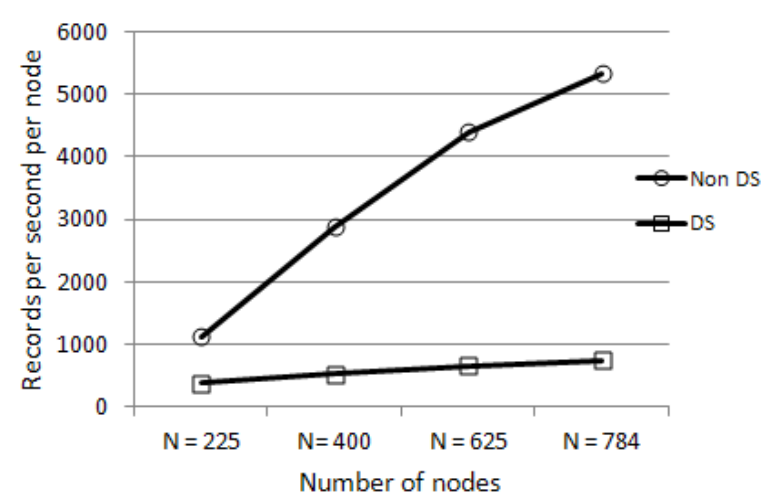

(a)

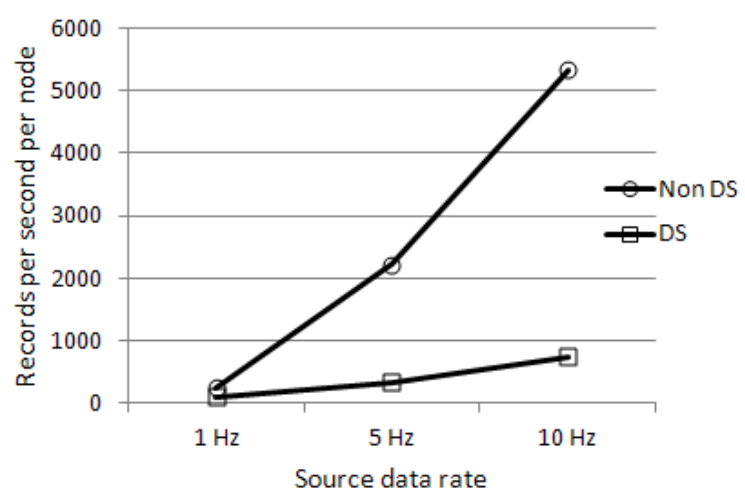

(b)

Fig. 12. Comparison of message complexity: VCAST vs non distance-sensitive forwarding. (a) Number of vehicular records transmitted per second per node at different network sizes for $p=10 \mathrm{~Hz}$ and range $100 \mathrm{~m}$. (b) Number of vehicular records transmitted per second per node at different source broadcast rates for $\mathbf{7 8 4}$ nodes and range $100 \mathrm{~m}$ 


\subsubsection{Impact of time varying inter-vehicular separations}

In a real traffic network vehicle density varies over time. There could be instants when a vehicle has lot of neighbors within communication range and also instances when there are no neighbors.

The aim of this subsection is to characterize the performance of VCAST in the presence of time varying inter-vehicular separations caused by non-uniform mobility patterns. Therefore, in this simulation, we have chosen the random $2 d$ walk mobility pattern with time varying speeds in the range of $20 \mathrm{~m} / \mathrm{s}$ to $40 \mathrm{~m} / \mathrm{s}$.

In Fig. 13(a) and Fig. 13(b), we compare the information staleness graphs for the random and uniform mobility patterns at $5 \mathrm{~Hz}$ and $10 \mathrm{~Hz}$ source broadcast rates with 784 nodes. As seen from these figures, the graphs are quite similar; highlighting that random mobility does not significantly impact the performance. Staleness values at larger distances are observed to be slightly higher for the case of random mobility. However, the reason for this is not higher communication cost but rather the fact that the severe random mobility scenario often creates sparse and disconnected regions within the network, thereby increasing the number of hops traversed between two vehicles at a given distance. This effect is more pronounced at larger distances.

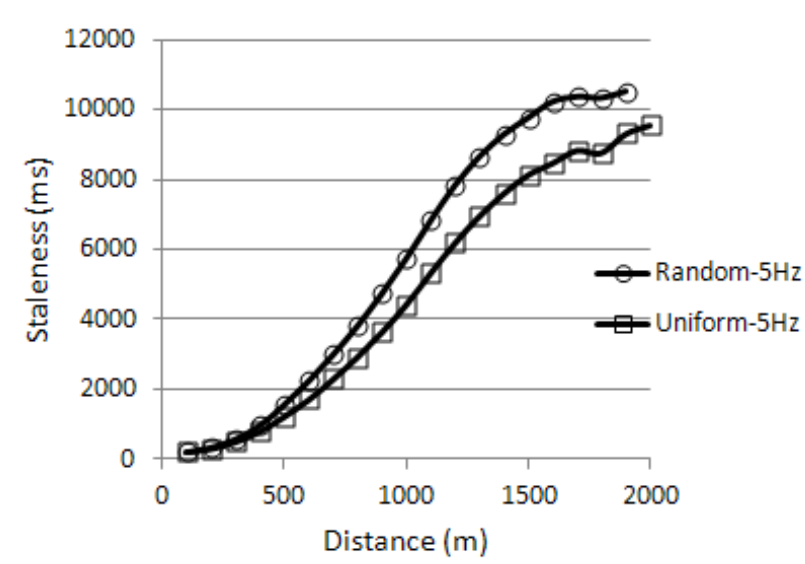

(a)

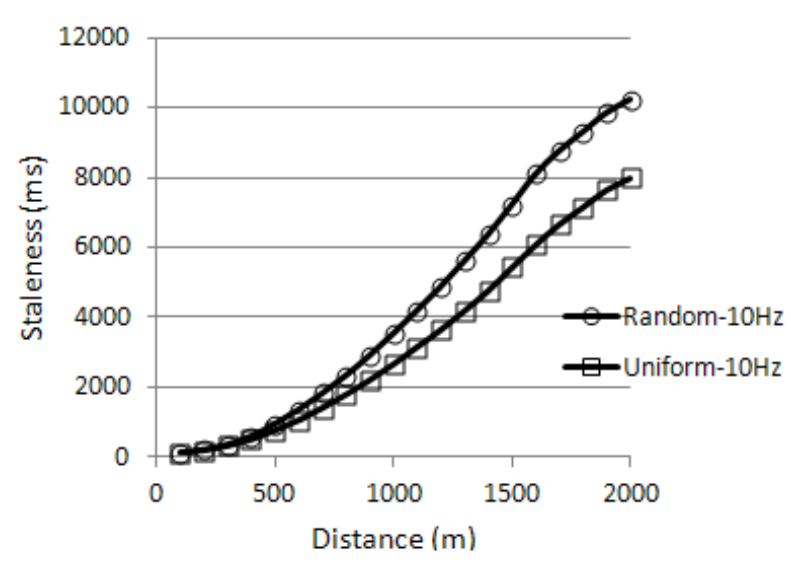

(b)

Fig. 13. Impact of time varying random mobility pattern on VCAST: (a) Maximum staleness vs pair-wise vehicular distance for uniform and random mobility with 784 vehicles, Communication range $100 \mathrm{~m}$, source broadcast rate $5 \mathrm{~Hz}$ (b) Maximum staleness vs pair-wise vehicular distance for uniform and random mobility with 784 vehicles, Communication range $100 \mathrm{~m}$, source broadcast rate $10 \mathrm{~Hz}$ 
In Fig. 14, we compare the average communication cost for the random and uniform mobility scenarios at two different source broadcast rate. This graph highlights the fact that communication costs do not increase in the random mobility scenario - in fact a small decrease is observed in the average communication cost over several random patterns.

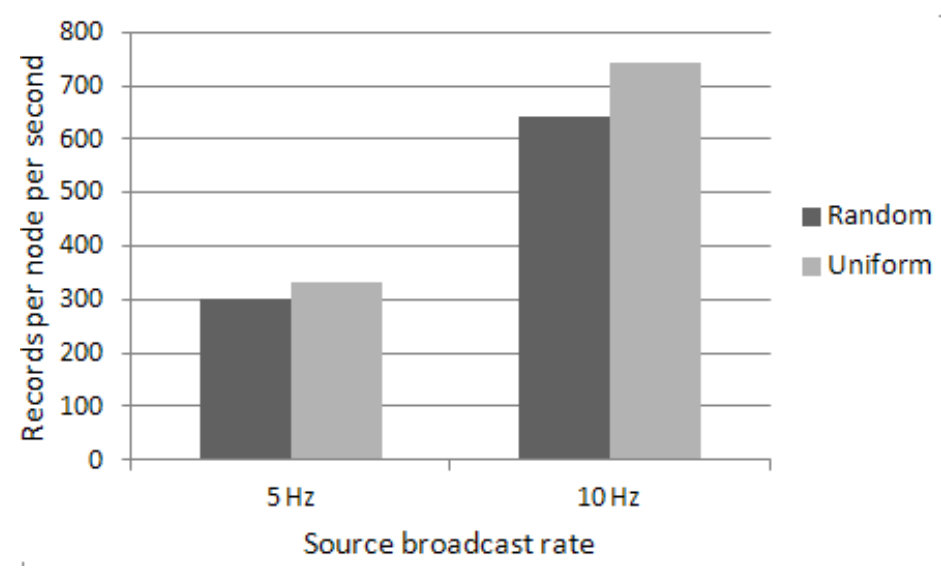

Fig. 14. Impact of time varying random mobility pattern on VCAST (comparison of communication cost): Number of vehicular records transmitted by each node per second at $5 \mathrm{~Hz}$ and $10 \mathrm{~Hz}$ source broadcast rates under uniform and random mobility patterns

\section{Analyzing The Impact of VCAST On Vehicular Safety}

In this section we study impact of multi-hop network on vehicle safety. String stability problems amplify only in large vehicle strings. It is very costly as well as time consuming to create strings of hundreds of vehicles in real world. So we use simulation to study this impact. There are two independent simulation components required for this study. First is network service component for connecting vehicles through wireless communication. This has been discussed above. Second is the vehicle movement engine. For this purpose we developed car following module in C++ entirely from scratch. This module extends NS3's mobility model framework. Note that NS3 does not provide native mobility model for simulating vehicle movement in road traffic. Our own implementation also gives us the freedom to play with simulation parameters. 


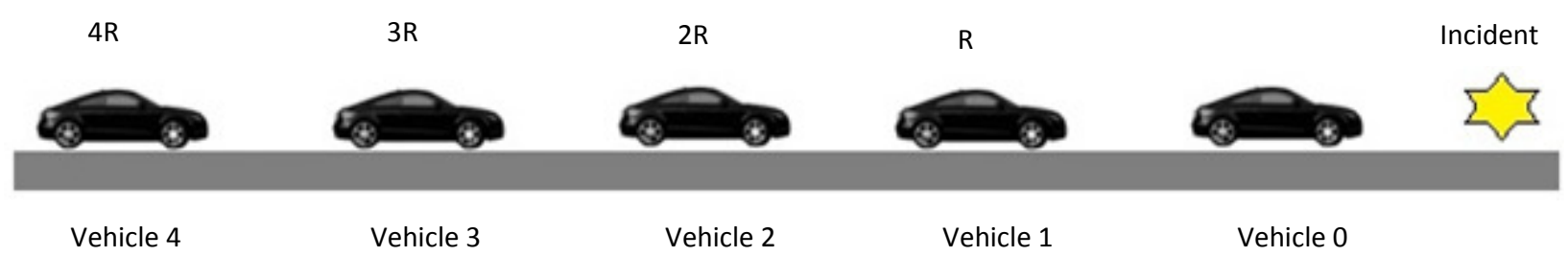

(a)

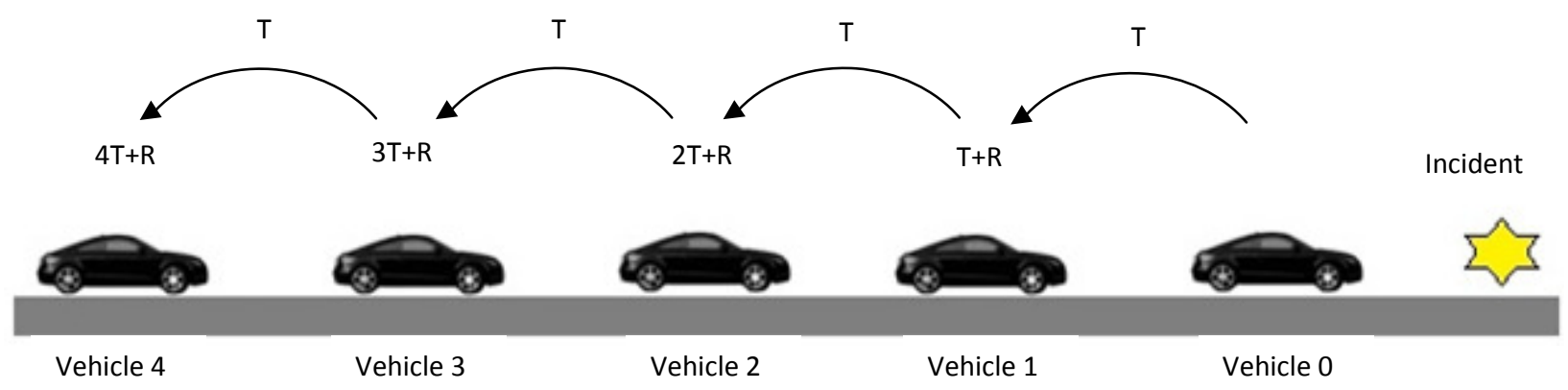

(b)

Fig. 15. Response of followers when lead vehicle 0 detects an incident (a) Vehicles are not in network and so the follower's reaction depend on its immediate predecessor. The reaction time gets staggered up which ultimately leads to collision for vehicles at the far end of the string. (b) Vehicles are in network. Network time gets staggered up instead of reaction time. Since network time $<$ reaction time, rear end collisions are avoided.

Let us consider a platoon of cars traveling in single lane in single direction. For simplicity of explanation, we will consider only five cars. Vehicle 0 is the lead vehicle which detects an incident and applies brakes, consequently switching his brake lights on. $\mathrm{R}$ is the time it takes each driver to realize that his predecessor's brake lights are on and he should also apply brakes. This reaction time R propagates up the vehicle string and starts to accumulate. As a result, cars further up the string are more likely to crash. If car 0 's brake lights are turned on at time $t=0$, car 1's driver gets warned of the slowdown at time $t=0$ but applies brakes at time $t=R$. Similarly, car 2's driver gets warned at time $t=R$ but applies brake at time $t=2 R$ and so on. In general, for kth car, driver receives warning at time $t=(k-1) R$ and applies brake at time $t=k R$.

If the vehicles are connected through wireless communication, leader car 0 sends a slow down message as soon as it applies brakes. Let us assume that a message takes t time to propagate from one car to another. 
Follower car does not have to depend on brake lights of the predecessor. Follower can start applying brakes as soon as it receives a slow down message. Car 0 applies brakes at time $t=0$, car 1 gets warned of slowdown at time $t=T$ but applies brakes at time $t=T+R$. Similarly, car 2 gets warned at $t=2 T$ but applies brakes at $2 T+R$. In general, for kth car driver receives warning at time $t=k T$ and applies brake at time $t=k T+R$. If we compare the time in both the cases at which kth car receives slowdown warning message, we find that $k T<<(k-1) R$ because $T<<R$. Hence multi-hop network allow drivers to get early notification of an impending danger which could prevent rear end collisions.

\subsection{Vehicle Movement Simulation}

Vehicle movement is simulated with the help of mathematical model. A good model takes into account traffic dynamics as well as human driving behavior. When modeling vehicle movement, three characteristic time constants influence the dynamics of traffic flow. First is the reaction time or explicit delay $R$, which is time to react to an incident. Second is the vehicle adaptation time or implicit delay or first order time constant $\tau$, which is the time taken by vehicle powertrain to respond to new desired velocity. Third is the update time $\Delta t$, which is the simulation interval at which new desired velocity is calculated.

Fig. 16 shows relation of all three time constants, assuming $\mathrm{R} / \Delta t$ is an integer value, which is not always true. These three time constants do not just exist in mathematical models. They are consistent with the real world observation and are present in both manual driving as well as automated driving. For manual driving, update time represents the length of time period where drivers do not draw attention to driving task and consequently do not adapt their speed according to the actual situation. For automated driving,

update time represents the sampling period of the sensors. Reaction time for manual driving is the time taken by drivers to react to a situation. Reaction time for automated driving is the sensing, processing and actuation delay. Velocity adaptation time represents vehicle powertrain delay in both the cases. This is 
because moving objects cannot respond to acceleration changes instantaneously. Hence all three time constants are essential in modeling vehicle motion.

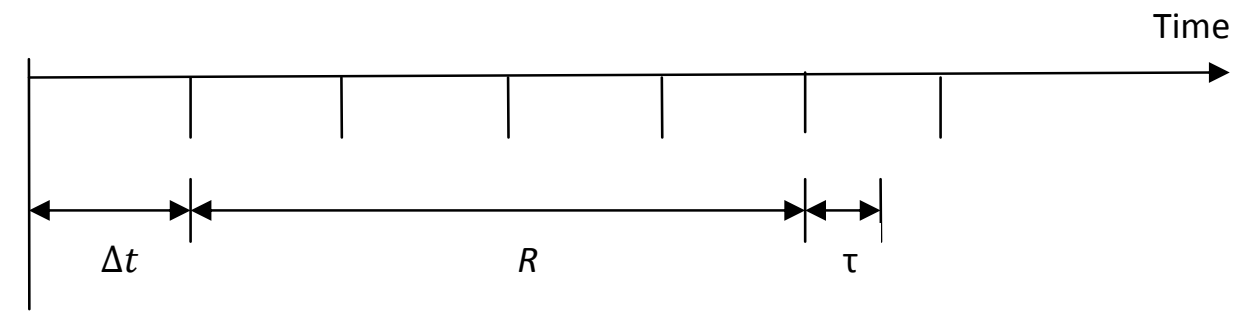

Fig. 16. Timing relation of Update time $\Delta t$, Reaction time $R$ and First order time constant $\tau$. These times have simple intuitive meaning. New response is computed at every $\Delta t$ time. It takes $R$ time to put it into action and after that it takes further $\tau$ time to complete the action.

\subsubsection{General Car Following Model}

Here and in the following, vehicle indices are ordered such that $i$ represents follower and $i-1$ represents predecessor. Vehicle distances are measured from start of the lane segment to the mid-point of vehicle's body. Distances are ordered such that $x_{i}$ represents follower's distance and $x_{i-1}$ represents predecessor's distance. Similarly, velocity vi represents follower's velocity and $\mathrm{v}_{\mathrm{i}-1}$ represents predecessor's velocity. $h$ is the headway time.

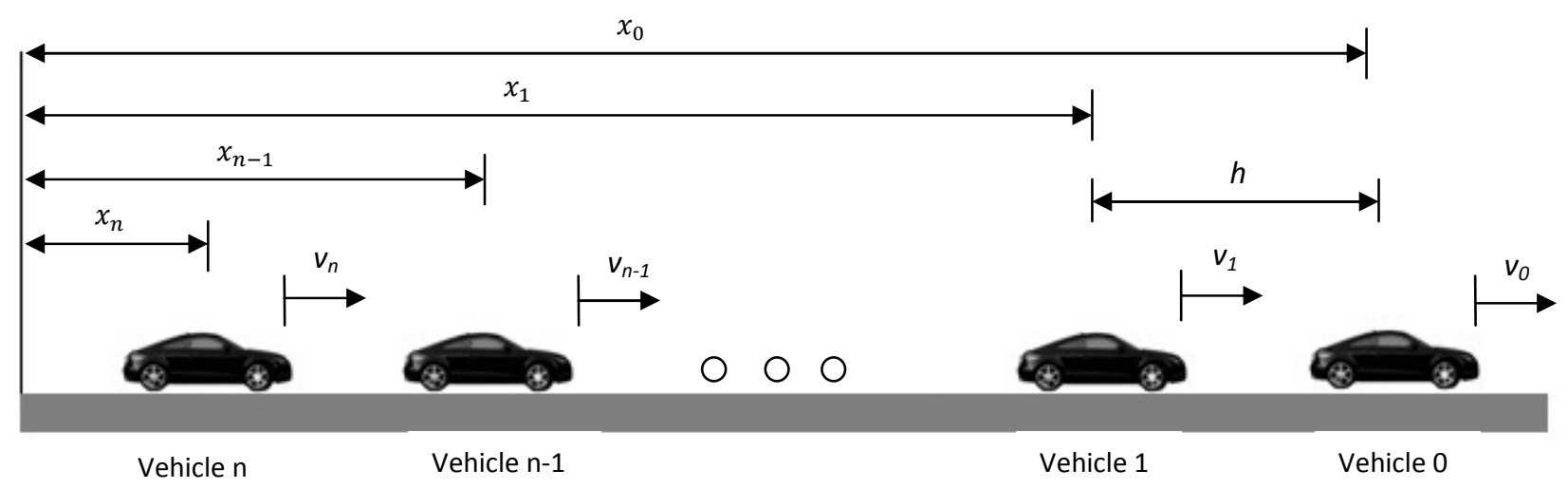

Fig. 17. Indexing scheme used in car following models; There are $\mathbf{n}$ vehicles in the platoon; Vehicle indices are $i=0,1,2,3, \ldots, n-1, n$ starting from the lead vehicle; Vehicles move in the direction shown by arrow with velocities $v_{0}, v_{1}, v_{2}, v_{3}, \ldots, v_{n-1}, v_{n}$ starting from the lead vehicle; Their respective distances from the lane start is $x_{0}, x_{1}, x_{2}, x_{3}, \ldots, x_{n-1}, x_{n} ; h$ is the constant headway time between followers and predecessors 
Most of the car-following models use the following acceleration update rule

$$
\frac{d v_{i}}{d t}=f\left(s_{i}, v_{i}, \Delta v_{i}\right)
$$

Here, acceleration depends upon its own velocity $v_{i}$, the inter vehicular gap $s_{i}$ and the velocity difference $\Delta v_{i}$. Gap is defined in terms of vehicle positions $x$ i.e. $s_{i}(t)=x_{i-1}(t)-x_{i}(t)$. Velocity difference is defined by $\Delta v_{i}=v_{i-1}-v_{i}$. This rule is time continuous. It is impossible to simulate in computers using this time continuous rule. It is solved by defining an update time interval $\Delta t$ within which acceleration is constant. This leads to the following update rules

$$
\begin{gathered}
v_{i}(t+\Delta t)=v_{i}(t)+\dot{v}_{i}(t) \Delta t \\
x_{i}(t+\Delta t)=x_{i}(t)+v_{i}(t) \Delta t+\frac{1}{2} \dot{v}_{i}(t)(\Delta t)^{2}
\end{gathered}
$$

For $\Delta t \rightarrow 0$, this scheme converges to the exact solution. Even though this update rule gives us approximate solution, update time $\Delta t$ has an intuitive value and so this update rule is widely used in simulations.

In vehicle motion modeling, velocity adaptation time is also called Implicit Delay and reaction time is called External Delay. Implicit delay is contained in the above equation and may not be directly modifiable. Example is Intelligent Driver Model (IDM) where implicit delay is contained in acceleration capability of the model. External delay is always modifiable and is external to the model.

\subsubsection{Car Following Model With First Order Dynamics}

This is the core of vehicle movement engine. In this thesis, we have simulated platoon of vehicles moving on a single lane in a single direction. This work can easily be extended for multiple lanes with lane changing vehicles. The choice of car following model was based on two criteria (1) Acceleration response should not be instantaneous (2) Model should give facility to manipulate Implicit and External Delay. Most of the car-following models fail on first criteria i.e. they make instantaneous adaptation of 
acceleration at discrete time. IDM is a very popular car following model but it fails on second criteria i.e. it does not give access to Implicit Delay parameter.

Best model we came across which satisfies both the criteria is a variation of Optimal Controller Model (OCM) with first order dynamics. The first order time constant is introduced to model the finite response of vehicle powertrain. Acceleration response in OCM for nth vehicle is given by

$$
a_{n}(t)=\alpha\left[\frac{x_{n-1}(t)-x_{n}(t)-D}{h}-v_{n}(t)\right]+k\left[v_{n-1}(t)-v_{n}(t)\right]
$$

where $D$ is the safe distance i.e. minimum distance at which follower must stop if the predecessor stops; $h$ is the headway time; $\alpha$ is the sensitivity; $k$ is the gain for feedback control.

Since the accelerations are not instantaneous, a more realistic equation with first order dynamics is given by

$$
\tau \frac{d a_{n}(t)}{d t}+a_{n}(t)=\alpha\left[\frac{x_{n-1}(t)-x_{n}(t)-D}{h}-v_{n}(t)\right]+k\left[v_{n-1}(t)-v_{n}(t)\right]-\xi a_{n}(t)
$$

where $\tau$ is the first order time constant; $\xi$ is acceleration feedback control. First order time constant determines how quickly a system responds to input. Approximately, first order time constant is the time it takes the system to achieve $63 \%$ of the input. Larger the value of time constant, slower is the response. A simple first order system can be represented by

$$
\tau \frac{d x}{d t}+x(t)=G u(t)
$$

where $x(t)=$ Response of the system, $u(t)=$ Input to the system, $\tau=$ First order time constant, $G=$ Gain of the system. For a step response, solving differential equation ( 5 ) gives us the following 


$$
x(t)=e^{\frac{-t}{\tau}}(x(0)-G u(t))+G u(t)
$$

Equation ( 6 ) is used to calculate the acceleration response of vehicles where

- $x(t)=a_{n}(t)$, the acceleration response

- $u(t)=\alpha\left[\frac{x_{n-1}(t)-x_{n}(t)-D}{h}-v_{n}(t)\right]+k\left[v_{n-1}(t)-v_{n}(t)\right]-\xi a_{n}(t)$, the input

- $x(0)=a(0)$, the starting acceleration in that update interval

If there is also a finite reaction time $R$, equation ( 6 ) becomes

$$
x(t+R)=e^{\frac{-t}{\tau}}(x(0)-G u(t))+G u(t)
$$

It means that due to reaction time, the acceleration response is delayed by $R$ time.

The simulation parameter values for the model are taken from [44].

\begin{tabular}{|l|l|l|}
\hline Parameter & Description & Value \\
\hline$\alpha$ & Sensitivity & $2 \mathrm{sec}^{-1}$ \\
\hline$K$ & Gain for the feedback control & $1 \mathrm{sec}^{-1}$ \\
\hline$\xi$ & Acceleration feedback control & $0.6,0.99$ \\
\hline$D$ & Safe stopping distance & 4 meters \\
\hline
\end{tabular}

Table 1. Simulation parameters for the chosen car following model which are used throughout this thesis, unless otherwise stated.

$a, x, v, h, R$ and $\tau$ are variables. String stability of platoon is controlled by variables $\mathrm{R}$ and $\tau$, as explained in the next section.

\subsection{String Stability Analysis}

For the car following model in ( 7 ), there exists an upper bound on reaction time $R$ which is a function of first order time constant $\tau$ [44]. Fig. 18 shows the upper bound for three different acceleration 
feedback control i.e. $\xi=0, \xi=0.6, \xi=0.99$. Any combination of $\tau$ and $R$ within the upper bound gives a string stable system. Any combination beyond the upper bound gives string unstable system. For example in Fig. $18,\left(R_{1}, \tau_{1}\right)$ gives string unstable system for all $\xi ;\left(R_{2}, \tau_{2}\right)$ gives string stable system for $\xi=0.99$ but string unstable system for $\xi=0, \xi=0.6$. Hence for a given $\xi$, string unstable system can be generated with careful choice or $R$ and $\tau$.

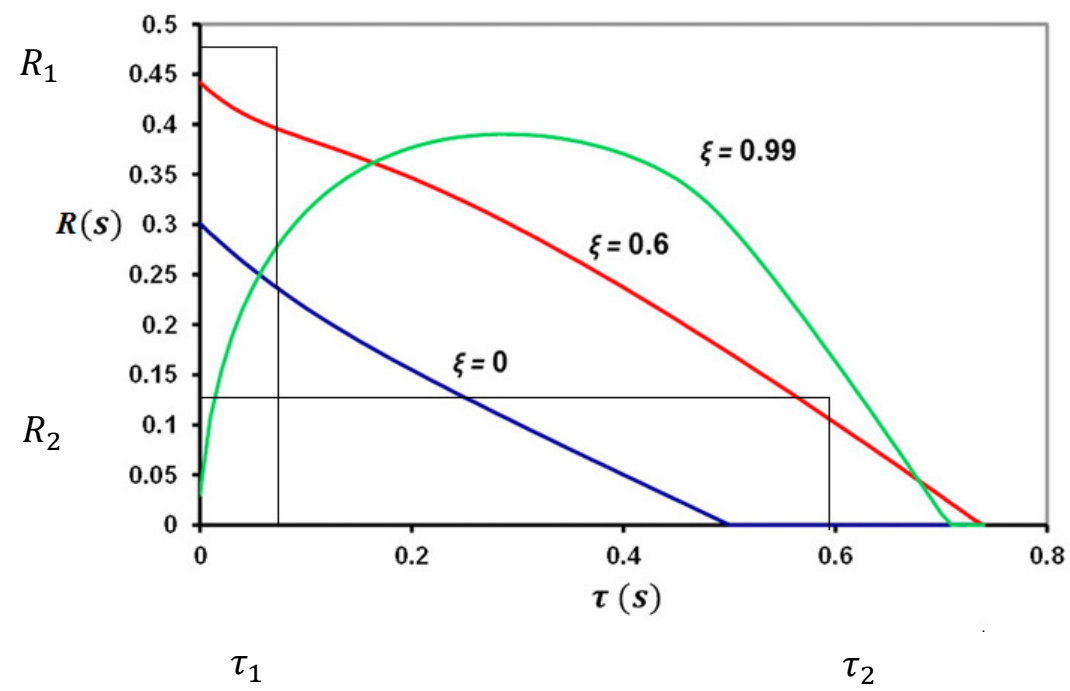

Fig. 18. The upper bound on $R$ as a function of $\tau$ for different acceleration feedback control $\xi$. Parameters $\alpha$ $=2 \mathrm{~s}^{-1}, \mathrm{k}=1 \mathrm{~s}^{-1}$ and $\mathrm{h}=1 \mathrm{~s} ;\left(R_{1}, \tau_{1}\right)$ and $\left(R_{2}, \tau_{2}\right)$ are any two arbitrary pair of values.

The string-stability ensures that perturbation decrease as they propagate up the vehicle stream. [47] defines perturbation and string stability in the following way.

Perturbation can be measured in terms of two errors

\section{- Range Error}

$$
\varepsilon_{i}=x_{i-1}-x_{i}-h_{i} v_{i}
$$

where $h_{i}$ is current headway time and $v_{i}$ is velocity of $\mathrm{i}^{\text {th }}$ vehicle.

\section{- Rate Error}

$$
\varepsilon_{v i}=v_{i-1}-v_{i}
$$


A vehicle string is string stable if the Euclidean norm of followers range/rate error is less than the Euclidean norm of predecessors range/rate error.

$$
\begin{gathered}
\left\|\varepsilon_{i+1}\right\|_{2} \leq\left\|\varepsilon_{i}\right\|_{2} \\
\left\|\varepsilon_{v i+1}\right\|_{2} \leq\left\|\varepsilon_{v i}\right\|_{2}
\end{gathered}
$$

In this thesis we will only take rate errors, unless otherwise stated. The results hold true for range error as well.

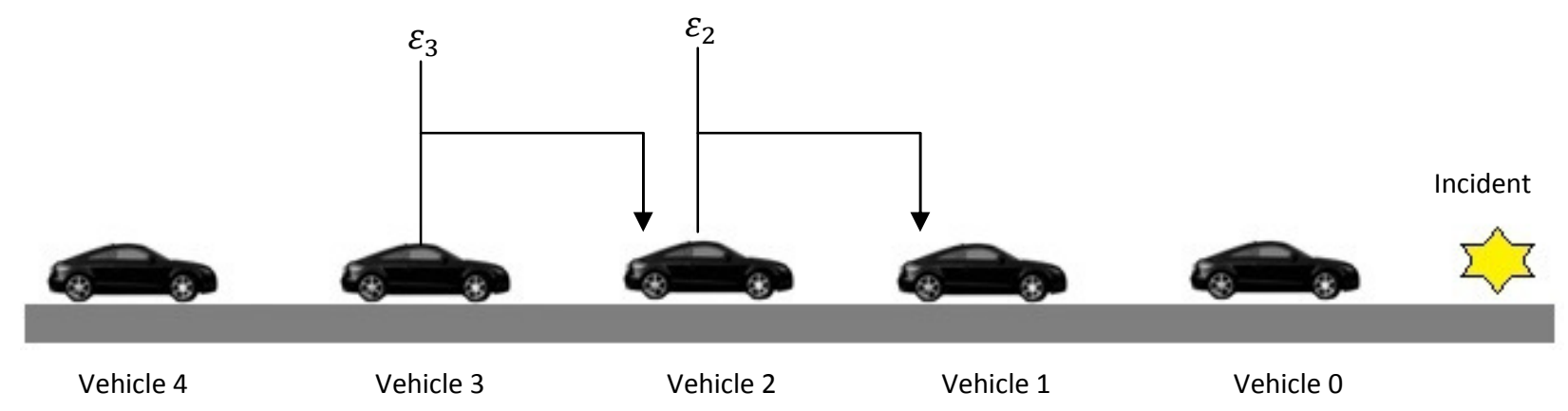

Fig. 19. Rate error of vehicle 2 is the velocity difference between vehicle 2 and vehicle 1 . Similarly rate error of vehicle 3 is velocity difference between vehicle 3 and vehicle 2 .

To calculate Euclidean norm, the rate error vectors are created by recording rate errors for the entire duration of simulation steps. For example, if there are total of $n$ simulation steps, then the rate error vector for vehicle 2 will be $\varepsilon_{2}^{0}, \varepsilon_{2}^{1}, \varepsilon_{2}^{2}, \varepsilon_{2}^{3}, \ldots, \varepsilon_{2}^{n}$; where subscript is the vehicle index and superscript is the simulation steps.

In general, for total of $n$ simulation steps, Euclidean norm for ith vehicle is

$$
\left\|\varepsilon_{i}\right\|_{2}=\sqrt{\left(\varepsilon_{i}^{0}\right)^{2}+\left(\varepsilon_{i}^{1}\right)^{2}+\left(\varepsilon_{i}^{2}\right)^{2}+\left(\varepsilon_{i}^{3}\right)^{2}+\ldots+\left(\varepsilon_{i}^{n}\right)^{2}}
$$


For string stability the Euclidean norm of each vehicle pair should decrease along the platoon. If we plot the Euclidean norm of each vehicle pair, a falling curve denotes string stability. Conversely, a rising curve denotes string instability.

\subsection{Dynamic headway adjustment}

In a multi-hop network, messages can propagate very quickly over large geographic distances. We have used this property to ensure no rear end collisions for VSS. When any moving vehicle detects an incident, it constructs a Collision Warning Message (CWM) and broadcasts this message to its follower. The vehicle that detects the incident is the leader. This CWM propagates up the vehicle stream hop by hop. Any follower receiving this CWM reacts by putting brakes and increasing its headway time according to the rule

$$
h_{i}=h_{\text {min }}+\frac{\left(\frac{x_{i-1}-x_{i}}{v_{i}}\right)}{h_{\min }}
$$

where $h_{i}$ is the headway for ith vehicle and $h_{\min }$ is the minimum headway for normal traffic. $h_{\min }$ is also the initial headway for all the vehicles at the start of simulation. In an equilibrium state, $h_{i}$ remains constant. When there is perturbation, $h_{i}$ increases to dampen the perturbation and approaches a stable value $h_{\text {stable }}$. This value $h_{\text {stable }}$ is the final headway time for vehicles to be string stable. $h_{\text {stable }}$ is greater than $h_{\min }$.

Since $h_{\text {stable }}$ is large, the traffic throughput decreases due to the increased headway. After the adjustment, the leader tries to find out if the traffic is stable at this headway. If it is stable, the leader again forwards a message to all its followers urging them to decrease their headway in such a way that string stability condition is satisfied throughout. The rule used is 


$$
\text { i.e. } \quad h_{i}=h_{\min }+K\left[\frac{\left(\frac{x_{i-1}-x_{i}}{v_{i}}\right)}{h_{\min }}\right]
$$

where $K$ is reduction factor $<1 . h_{i}$ approaches the minimum headway for normal traffic $h_{\text {stable }}^{\prime}$ while maintaining string stability. $h_{\text {stable }}^{\prime}$ is less than $h_{\text {stable }}$.

\subsection{Results}

\subsubsection{Measurement Strategy}

To investigate the impact of multi-hop forwarding in vehicle safety, we implemented (1) Car following model for simulating vehicle motion (2) VCAST like multi-hop network abstraction to simulate multi-hop network forwarding environment. Network abstraction is not required if the module is plugged into Ns3. To show the impact of multi-hop network on vehicle safety, we compare the results of vehicle platoon having multi-hop network with vehicle platoon not having multi-hop network. Vehicle safety is measured by introducing a perturbation in an already stable traffic flow and observing whether the perturbation increases or decreases up the traffic stream. If the perturbation increases, the platoon is assumed to be string unstable and thus is not safe. Otherwise, it is safe. We further show that the safety is achieved by dynamically adjusting headway time. Headway time adjustment makes vehicle platoon string stable in both the cases (1) When incident is detected and followers need to slow down to adjust to leaders speed (2) When resuming normal traffic once the traffic is stable and followers need to speed up to reduce the headway time

\subsubsection{Network Model}

We implemented VCAST like multi-hop network abstraction to speed up the simulation running times. The module is designed in such a way that replacing the abstraction with actual VCAST implementation in Ns3 won't have any compatibility issues. The abstraction gives facility for fully connected network (ITS), clusters of connected and unconnected network (ITS and manual driving) and fully switched off 
network (manual driving). The end to end message propagation time in each hop is assumed to be 0.1 seconds.

\subsubsection{Simulation Setup}

We have simulated a platoon of 100 vehicles moving in a single lane following a leader with externally controlled acceleration. The total run time of simulation is 500 seconds. At the start of simulation all the vehicles are in equilibrium state. Equilibrium state is defined when velocities of all the followers are equal to leader's velocity $v_{\text {lead }}=30 \mathrm{~ms}^{-1}$, accelerations of all the vehicles are zero i.e. $a=0$, headway times of all the followers are equal to $h_{\text {init }}=2$ secs and inter-vehicular gaps of all the followers are equal to $s_{e}=\left(v_{\text {lead }} * h_{\text {init }}\right)+s_{0}$, where $s_{0}$ is the minimum stopping distance. To trigger perturbation, the externally controlled vehicle i.e. the leader is decelerated from $30 \mathrm{~ms}-1$ to $20 \mathrm{~ms}-1$ after 50 seconds of simulation time. This is done by supplying deceleration a $=-2 \mathrm{~ms}-2$ for 5 seconds during time interval 50 secs $<=\mathrm{t}<=54$ secs. The leader then drives at constant velocity $v_{\text {lead }}{ }^{\prime}=20 \mathrm{~ms}^{-1}$ afterwards.

We have used the simulation parameters as listed in Table 1. First order reaction time is fixed at $\tau=0.1$ secs. This is reasonable value for vehicle powertrain delay. We have taken best case reaction time $\mathrm{R}=0.3$ secs and worst case reaction time $\mathrm{R}=0.7$ secs. Lower reaction times are not representative of the real world situation. There is no guarantee of lower reaction time due to various factors like limited capability of equipments, equipment failures etc. Best case reaction time is given only for the sake of comparison.

\subsubsection{Impact Without Multi-Hop Network}

\subsection{Small Reaction Time $\mathrm{R}=\mathbf{0} .3$ secs}

First we show impact of vehicle safety when reaction time is very low. Fig. 20(a) shows velocity profile of every tenth vehicle in a platoon of 100 cars. First profile is that of the externally controlled leader. After 50 seconds into simulation, the leader decelerates constantly at a $=-2 \mathrm{~ms}^{-2}$ for 5 seconds i.e. its velocity falls from $30 \mathrm{~ms}^{-1}$ to $20 \mathrm{~ms}^{-1}$. To avoid collision, all the followers decrease their velocities and settle at $20 \mathrm{~ms}^{-1}$. The time lag in velocity reduction of all the followers is equal to the reaction time $\mathrm{R}$ 
$=0.3$ secs. Note that Fig. 20 (a) shows larger than 0.3 secs time lag because it is the velocity profile of every tenth vehicle. Fig. 20 (b) shows inter car gap profile of every tenth vehicle pair. Initially all vehicles have equilibrium gap $s_{e}=65 \mathrm{~m}$ approx. After 50 seconds into simulation, the leader decelerates constantly for 5 seconds and the inter car gap between leader and first follower falls from $65 \mathrm{~m}$ to $45 \mathrm{~m}$ approx. This in turn triggers inter car gap of all the follower vehicles to fall from $65 \mathrm{~m}$ to $45 \mathrm{~m}$. The time lag is again equal to the reaction time $\mathrm{R}=0.3$ secs. Fig. 20 (c) shows Euclidean norm of the rate error. The platoon is string stable at $\mathrm{R}=0.3$ secs. This is because the reaction time is too less to cause any significant impact on string stability.

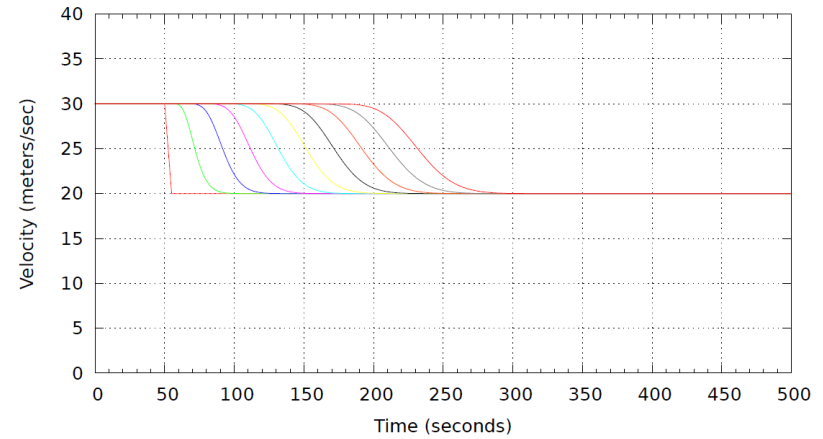

(a)

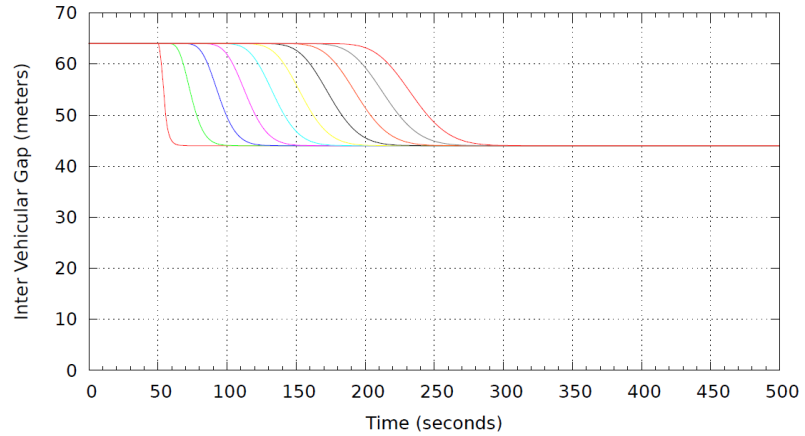

(b)

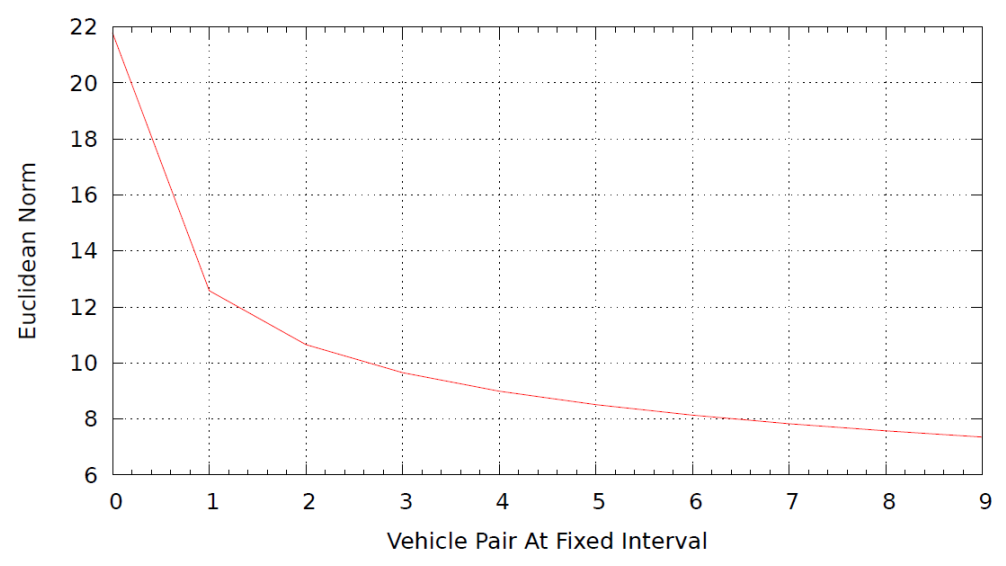

(c)

Fig. 20. Vehicle platoon without multi-hop network and small reaction time $R=0.3$, constants are taken from Table 1 (a) Velocity profile of leader and every tenth follower (b) Inter car gap profile of lead pair and every tenth vehicle pair (c) Euclidean norm plot of lead pair and every tenth vehicle pair. 


\subsection{Large Reaction Time $\mathrm{R}=0.7$ secs}

For larger reaction time of 0.7 secs, we see continuous oscillations for velocity and inter car gap profiles refer Fig. 21 (a) and Fig. 21 (b). The velocity and inter car gap oscillations do not fade and remains constant. This causes stop and go traffic waves. If we look at the stability graph Fig. 21 (c), we see that the curve is rising steadily up the vehicle stream. This indicates that perturbation is magnified and the traffic is string unstable.

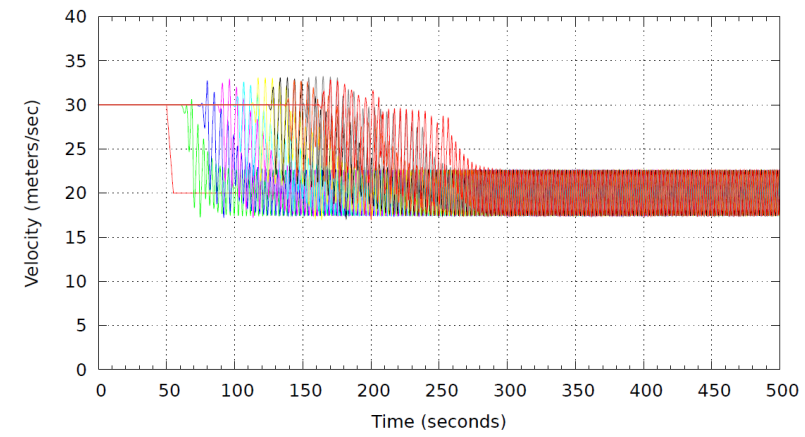

(a)

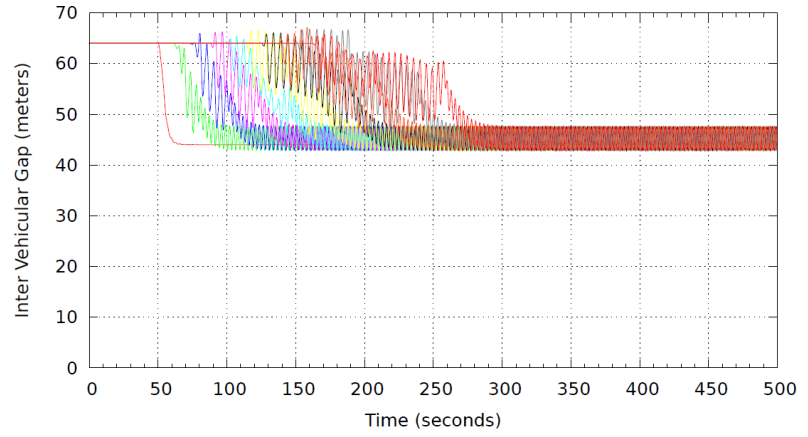

(b)

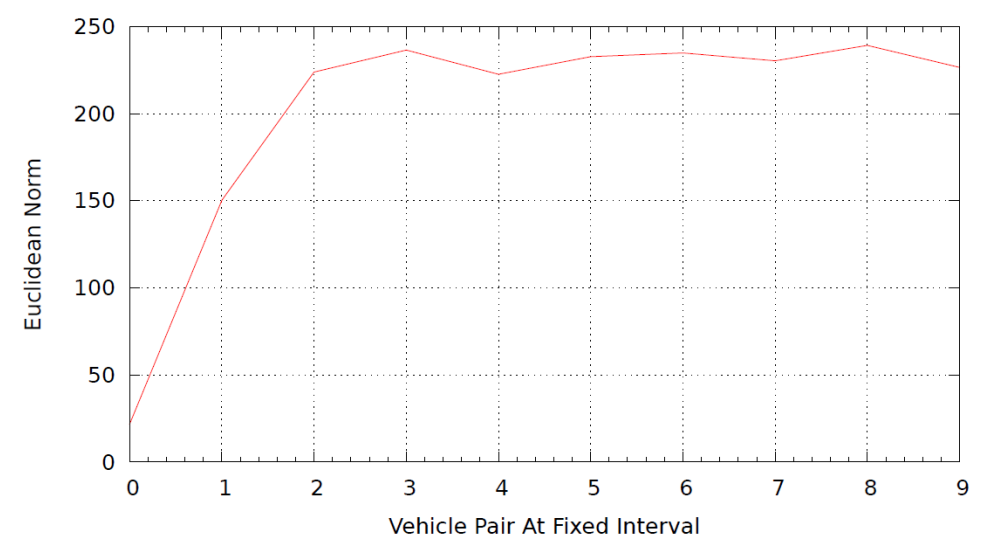

(c)

Fig. 21. Vehicle platoon without multi-hop network and large reaction time $R=0.7$, constants are taken from Table 1 (a) Velocity profile of leader and every tenth follower (b) Inter car gap profile of lead pair and every tenth vehicle pair (c) Euclidean norm plot of lead pair and every tenth vehicle pair.

Here is the zoomed in version of velocity profile and inter car gap profile to show the nature of oscillations. 


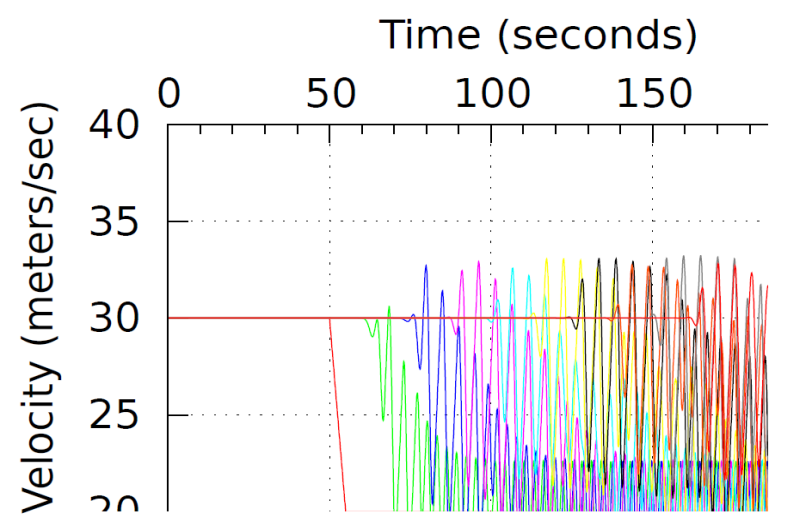

(a)

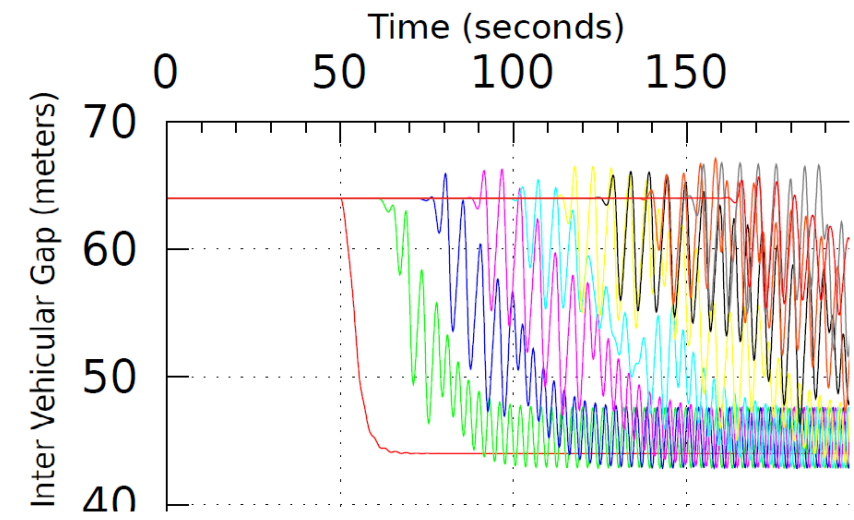

(b)

Fig. 22. Zoomed in profiles (a) Velocity profile zoomed in (b) Inter vehicle gap profile zoomed in

\subsubsection{Impact with multi-hop network}

\subsection{Incident Detected}

Fig. 23 shows the plots for a platoon of vehicles all connected in a multi-hop network. The sudden spike of headway time in Fig. 23 (c) is due to safety message received by all the followers. For followers to increase headway, they have to put brakes. Hence their speed decreases as shown in Fig. 23 (a). Also, their inter-car gap increases due to the speed reduction, which is shown in Fig. 23 (b).

Headway time depends on velocity of vehicle and inter-car gap of the vehicle with its leader. So headway time stabilizes when the velocity and inter-car gap stabilizes. The final stable value of headway time also depends on final stable values of velocity and inter-car gap. Stability plot Fig. 23 (d) shows that the traffic is string stable. 


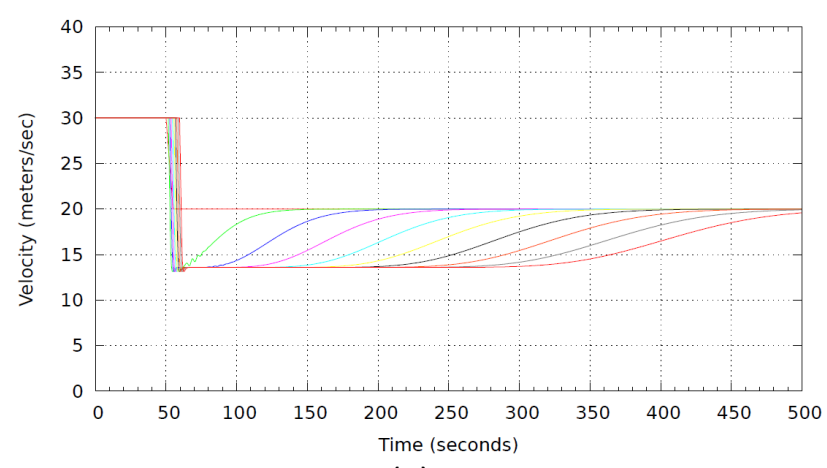

(a)

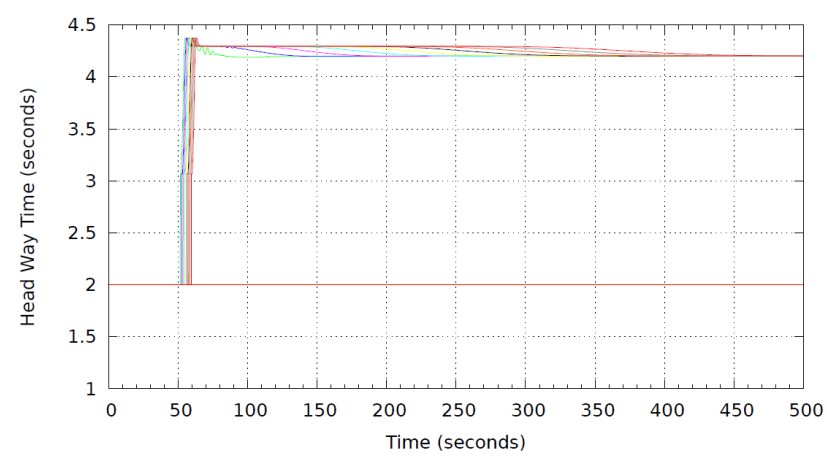

(c)

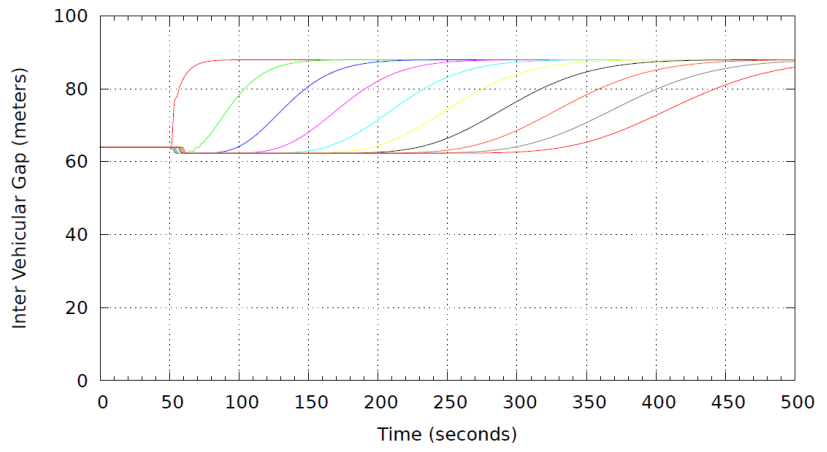

(b)

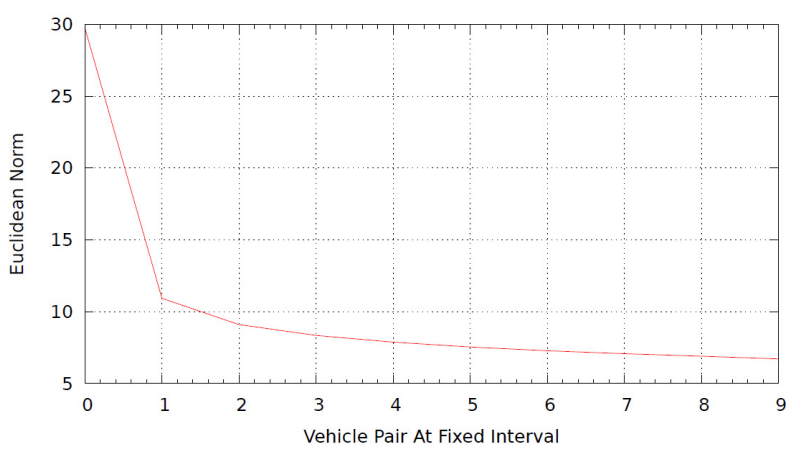

(d)

Fig. 23. Vehicle platoon in multi-hop network when incident detected. Reaction time $R=0.7$, constants are taken from Table 1. (a) Velocity profile of leader and every tenth follower (b) Inter car gap profile of lead pair and every tenth vehicle pair (c) Headway time profile of leader and every tenth follower (d) Euclidean norm plot of lead pair and every tenth vehicle pair.

Next, we repeat the experiment with the same platoon of vehicles, see Fig. 24. But this time the first five vehicles are not in network and rest are in network. It means that for the first five vehicles, there are no safety messages and they rely on predecessor's reaction time. Sixth vehicle acts as the leader and generates safety message which gets propagated to its followers hop by hop. The vehicle string is stable even in this case as shown by Fig. 24 (d). 


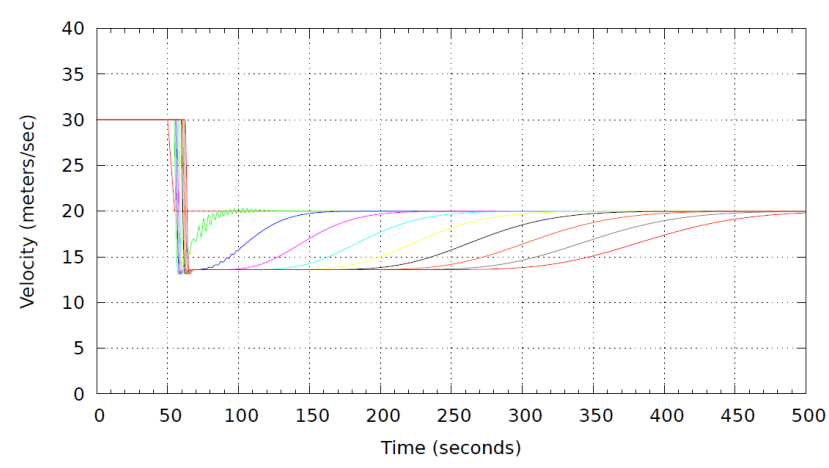

(a)

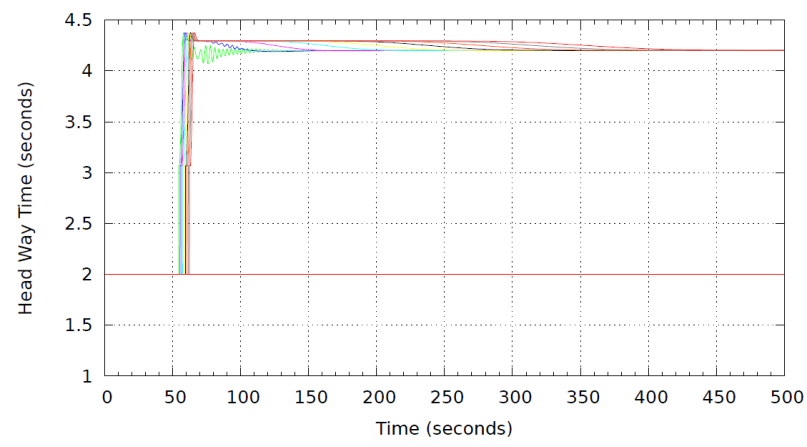

(c)

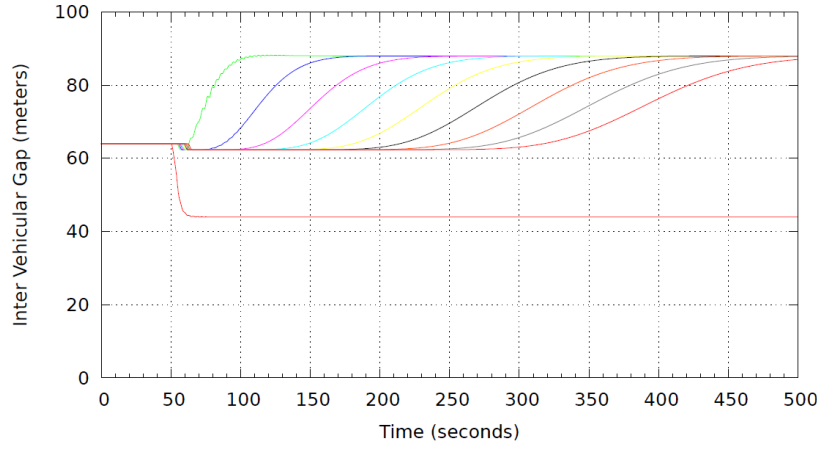

(b)

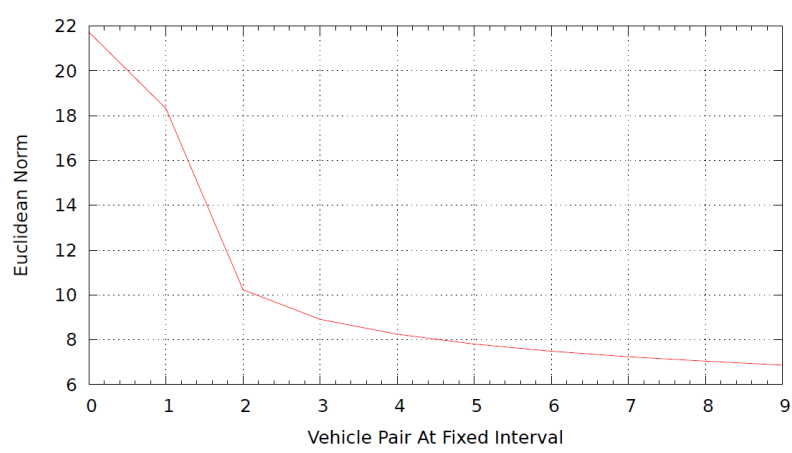

(d)

Fig. 24. All but first five vehicles are in multi-hop network when incident detected. Reaction time $\boldsymbol{R}=\mathbf{0 . 7}$, constants are taken from Table 1. Upon detection of incident, the leader which is the sixth vehicle sends safety messages to its followers which are forwarded hop by hop to the entire platoon (a) Velocity profile of leader and every tenth follower (b) Inter car gap profile of lead pair and every tenth vehicle pair (c) Headway time profile of leader and every tenth follower (d) Euclidean norm plot of lead pair and every tenth vehicle pair.

\subsection{Resume normal traffic}

After the leader detects the traffic is stable, it again sends special message to its followers urging them to reduce their headway, as shown in Fig. 25. In order to reduce headway, the followers increase their velocity as shown in Fig. 25 (a) as a result of which the inter vehicle distances decreases, as shown in Fig. 25 (b). After the desired headway is achieved, the followers decrease their velocity Fig. 25 (a) and travel at this reduced headway. The headway time approaches the minimum stable headway at which the traffic throughput is maximum Fig. 25(c). The decrease of headway happens in a string stable manner, as shown 
by Fig. 25 (d). It means the string stability condition is satisfied while the headway is being reduced, ensuring vehicle safety from rear end collisions while resuming normal traffic flow.

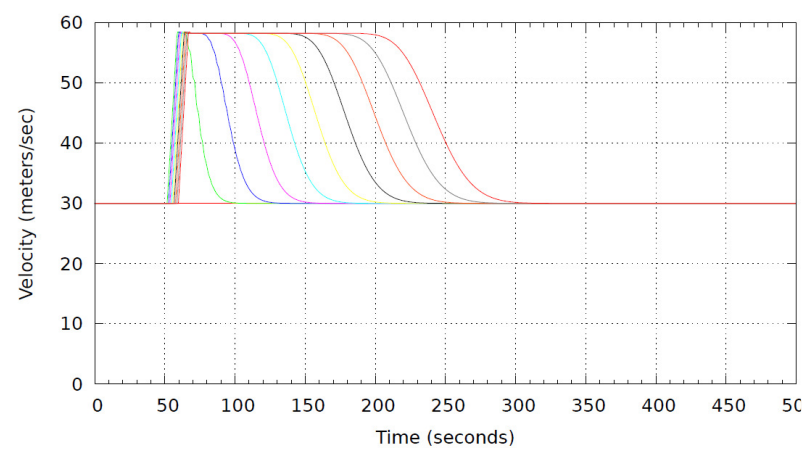

(a)

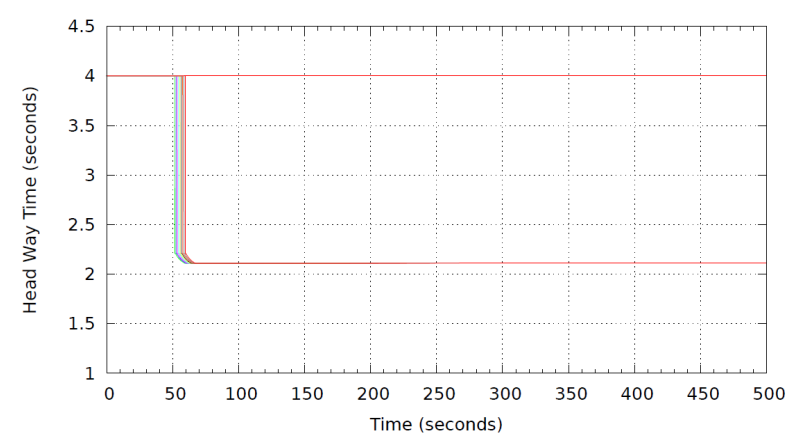

(c)

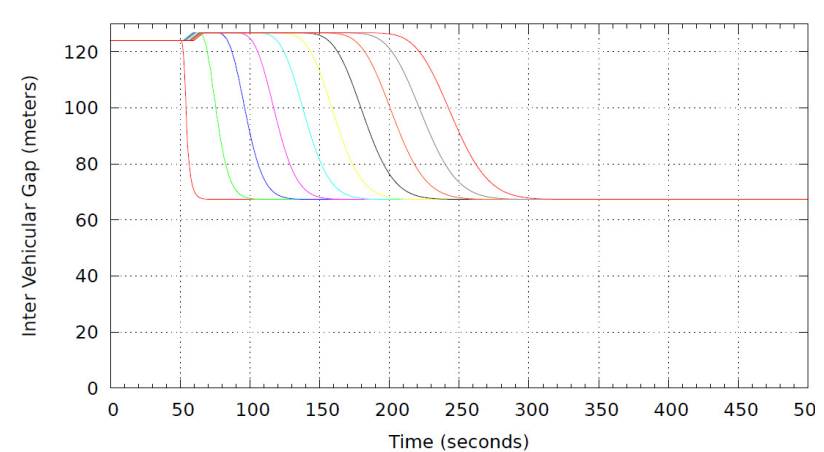

(b)

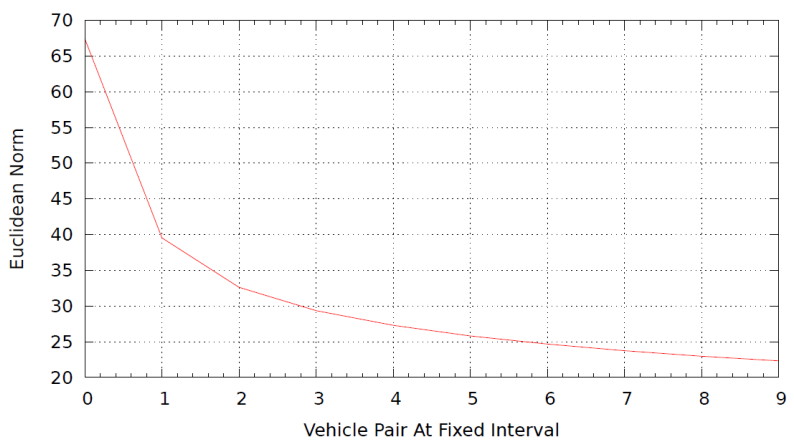

(d)

Fig. 25. Vehicle platoon in multi-hop network when resuming normal traffic. Reaction time $\boldsymbol{R}=\mathbf{0 . 7}$, constants are taken from Table 1. (a) Velocity profile of leader and every tenth follower (b) Inter car gap profile of lead pair and every tenth vehicle pair (c) Headway time profile of leader and every tenth follower (d) Euclidean norm plot of lead pair and every tenth vehicle pair.

\section{Conclusion and Future work}

In this thesis, we analyzed how multi-hop vehicular networks impact Vehicle Safety Applications. As this analysis could not be conducted physically in real world traffic due to limitation of time and budget, we took the help of simulation. The study is particularly challenging because it requires network simulation and traffic simulation in a tight loop, one impacting the other. Network simulation is required for simulating wireless vehicle communication service and traffic simulation is required for simulating 
vehicle motion in realistic traffic scenario. There is no standard tool or software which couples these two simulation components. Hence the study is conducted in two separate but related parts.

In the first part, we measured the impact of wireless multi-hop network on vehicle traffic. In this part more emphasis was given to evaluating the multi-hop network service and less emphasis to realistic vehicle movement in traffic. For generating traffic network, we used $2 \mathrm{D}$ grid network and random $2 \mathrm{D}$ walk mobility model. VCAST was chosen as the multi-hop broadcasting service because it is able to supply timely information over large distances without compromising on data supply rates at smaller distances. In other words it satisfies two conflicting constraints for vehicle safety applications (1) acquire real-time information from immediate neighbors to react quickly to emergencies (2) acquire timely information from vehicles at large distances to plan actions in advance. VCAST achieves this by using distance sensitive information propagation technique, in which information is forwarded at a rate that decreases linearly with distance from the source. VCAST was evaluated by using extensive simulations in ns-3 under different density, source broadcast rates and communication range. The results showed that VCAST was scalable for - large number of vehicles and large source broadcast rates. It was further shown that successful scaling is achieved by reduced number of vehicle records transmitted per second per vehicle for varying network sizes and varying source broadcast rates.

In the second part, we measured the impact of wireless multi-hop network on vehicle safety. Since we were measuring vehicle safety more emphasis was given to realistic vehicle movement in traffic and less emphasis on multi-hop network service. For simulating multi-hop network service, we built a VCAST like multi-hop forwarding network abstraction. For simulating vehicle movement, we implemented a realistic car following model with first order dynamics where we can manipulate both implicit delay as well as external delay along with many other traffic parameters. Vehicle safety was measured in terms of string stability of vehicle platoons. We also proposed a proactive method of planning - and that is by controlling the headway time. Through extensive simulations, we showed that multi-hop network makes 
the entire platoon string stable when emergency road incidents are detected abruptly on its course. We further showed that multi-hop network can be used to reduce headway and bring traffic to normal flow, all the while maintaining string stability.

In future work, we would like to design technique that would enable vehicles to determine whether the traffic contains perturbation or not. This would be done using multi-hop network service. This technique would dictate when to reduce the headway time and bring vehicles to normal traffic flow. We would also like to analyze the impact of multi hop network in vehicle safety in the presence of multiple perturbations on the course. 


\section{References}

[1] Y. Fallah, C. Huang, R. Sengupta, and H. Krishnan. Design of cooperative vehicle safety systems based on tight coupling of communication, computing and physical vehicle dynamics. In ACM/IEEE International Conference on Cyber-Physical Systems, 2010.

[2] U.S. Department of Transportation. Achieving the Vision: From VII to IntelliDrive. Policy white paper, 2010.

[3] V. Kulathumani and Y. Fallah. Vcast: An infrastructure-less vehicular traffic information service with distance sensitive precision. In IEEE Vehicular Technology Conference (VTC), 2012.

[4] Y. Liu and F. Dion. Safety assessment of information delays on intelligent vehicle control system performance. In Proceedings of Transportation Research Board Conference, 2006.

[5] V. Kulathumani and A. Arora. Aspects of distance sensitive design of wireless sensor networks. In IEEE Workshop on Spatial Computing, 2008.

[6] V. Kulathumani, A. Arora, and S. Ramagiri. Pursuit control over wireless sensor networks using distance sensitivity properties. IEEE Transactions on Automatic Control, 56(10):2473-2478, 2011.

[7] F. Li. Routing in vehicular ad hoc networks: A survey. IEEE Vehicular Technology Magazine, 2(2):12-22, 2007.

[8] K. C. Lee, U. Lee, and M. Gerla. Survey of routing protocols in vehicular ad hoc networks. In Advances in Vehicular Ad-Hoc Networks:Developments and Challenges, pages 149-170. IGI-Global, 2009.

[9] Y. Lin, Y. Chen, and S. Lee. Routing protocols in vehicular ad hoc networks: A survey and future perspectives. Journal of Information Science and Engineering, 26(2):913-932, 2010.

[10] J. Zhao and G. Cao. VADD: vehicle-assisted data delivery in vehicular ad hoc networks. In IEEE International Conference on Computer Communications, 2006.

[11] C. Lochert, M. Mauve, H. Fera, and H. Hartenstein. Geographic routing in city scenarios. ACM SIGMOBILE Mobile Computing and Communications Review, 9(1):69-72, 2005.

[12] Y. S. Chen, Y. W. Lin, and C. Y. Pan. A diagonal-intersection-based routing protocol for urban vehicular ad hoc networks. Telecommunication systems, 46:299-316, 2010.

[13] V. Naumov and T. Gross. Connectivity-aware routing (CAR) in vehicular ad hoc networks. In IEEE International Conference on Computer Communications, 2007.

[14] T. Taleb, E. Sakhaee, A. Jamalipour, K. Hashimoto, N. Kato, and Y. Nemoto. A stable routing protocol to support its services in vanet networks. IEEE Transactions on Vehicular Technology, 56(6):3337-3347, 2007.

[15] W. Sun, H. Yamaguchi, K. Yukimasa, and S. Kusumoto. GVGrid: A QoS routing protocol for vehicular ad hoc networks. In IEEE International Workshop on Quality of Service, 2006.

[16] A. Skordylis and N. Trigoni. Delay-bounded routing in vehicular ad-hoc networks. In ACM International Symposium on Mobile Ad hoc Networking and Computing, 2008.

[17] H. P. Joshi, M. Sichitiu, and M. Kihl. Distributed robust geocast multicast routing for inter-vehicle communication. In WEIRD Workshop on WiMax, Wireless and Mobility, 2007.

[18] H. Lu and C. Poellabauer. Balancing broadcast reliability and transmission range in VANETs. In IEEE Vehicular Networking Conference (VNC), 2010.

[19] M. Torrent-Moreno, J. Mittag, P. Santi, and H. Hartenstein. Vehicle-to-vehicle communication: Fair transmit power control for safetycritical information. IEEE Transactions on Vehicular Technology, 58(7):3684 -3703, 2009.

[20] L. Cheng and R. Shakya. VANET adaptive power control from realistic propagation and traffic modeling. In IEEE conference on Radio and wireless symposium, 2010.

[21] L. Yang, J. Guo, and Y. Wu. Channel Adaptive One Hop Broadcasting for VANETs. In IEEE Conference on Intelligent Transportation Systems, 2008.

[22] C. Chigan and J. Li. A Delay-Bounded Dynamic Interactive Power Control Algorithm for VANETs. In IEEE International Conference on Communications, 2007.

[23] A. Bachir and A. Benslimane. A multicast protocol in ad hoc networks inter-vehicle geocast. In IEEE Semiannual Vehicular Technology Conference, 2003.

[24] Y. S. Chen, Y. W. Lin, and S. L. Lee. A mobicast routing protocol for vehicular ad hoc networks. ACM/Springer Mobile Networksand Applications, 15(1):20-35, 2010.

[25] T. Osafune, L. Lin, and M. Lenardi. Multi-hop vehicular broadcast (MHVB). In International Conference on Intelligent Transportation Systems telecommunications (ITST), 2006.

[26] J. Fukuyama. A probabilistic protocol for multi-hop routing in VANETs. In IEEE International Conference on Communications Workshop, 2009.

[27] D. Kumar, A. A. Kherani, and E. Altman. Route lifetime based optimal hop selection in VANETs on highway: an analytical viewpoint. In International IFIP-TC6 Networking Conference, 2006.

[28] T. Fukuhara, T. Warabino, T. Ohseki, K. Saito, K. Sugiyama, T. Nishida, and K. Eguchi. Broadcast methods for intervehicle communications system. In IEEE Wireless Communications and Networking Conference, 2005.

[29] F. Bai, D. Stancil, and H. Krishnan. Toward understanding characteristics of dedicated short range communications (dsrc) from a perspective of vehicular network engineers. In International Conference on Mobile Computing and Networking, MobiCom, 2010.

[30] S. Ni, Y. Tseng, Y. Chen, and J. Sheu. The broadcast storm problem in a mobile ad hoc network. In ACM/IEEE 
international conference on Mobile computing and networking, 1999.

[31] B. Williams and T. Camp. Comparison of Broadcasting Techniques for Mobile Ad Hoc Networks. In ACM International Symposium on Mobile Ad Hoc Networking and Computing (MobiHoc), 2002.

[32] L. Peterson and B. Davie. Computer networking: A systems approach. Elsevier, 2011.

[33] G. Pei, M. Gerla, and T.-W. Chen. Fisheye State Routing in Mobile Adhoc Networks. In ICDCS Workshop on Wireless Networks, pages 71-78, 2000.

[34] F. Dehne, A. Ferreira, and A. Rau-Chaplin. Parallel fractional cascading on hypercube multiprocessors. In Computational Geometry Theory Applications, volume 2, pages 144-167, 1992.

[35] S. Funke, L. Guibas, and Y. Zhang. Distance Sensitive Information Brokerage in Sensor Networks. In International Conference on Distrbuted Computing in Sensor Systems (DCOSS), pages 234-251. Springer-Verlag, 2006.

[36] M. Demirbas, A. Arora, and V. Kulathumani. Glance: A lightweight querying service for wireless sensor networks. Theoretical Computer Science, 410, February 2009.

[37] V. Kulathumani, Anish A. Arora, M. Sridharan, and M. Demirbas. Trail: A distance-sensitive sensor network service for distributed object tracking. ACM Transactions on Sensor Networks, 5(2):1-40, 2009.

[38] J. Gao, L.J. Guibas, J. Hershberger, and L. Zhang. Fractionally cascaded information in a sensor network. In IPSN, pages 311-319, 2004.

[39] V. Kulathumani and A. Arora. Distance sensitive snapshots in wireless sensor networks. In Principles of Distributed Systems (OPODIS), volume 4878, pages 143-158, 2007.

[40] E. W. Dijkstra. Self-stabilizing systems in spite of distributed control. Communications of the ACM, 17(11), 1974.

[41] Sheikholeslam, S. and Desoer, C. A., Longitudinal Control of a Platoon of Vehicles, Proc. of 1990 American Control Conference, San Diego, pp. 291-296, 1990.

[42] YanaKiev, D., Kanellakopoulos, I., A Simplified Framework for String Stability Analysis in AHS, Proc. of the 13th IFAC World Congress, Volume Q, pp.177-182, 1996.

[43] Swaroop, D., Hedrick, J.K., String Stability of Interconnected Systems, IEEE Trans. Automat. Contr., vol. 41, no. 3, pp. 349-356, 1996.

[44] L. C. Davis, Stability of adaptive cruise control systems taking account of vehicle response time and delay, Physics Letters A 376 2658-2662, 2012

[45] Animesh Chakravarthy, Kyungyeol Song, Eric Feron, Preventing Automotive Pileup Crashes in Mixed-Communication Environments, IEEE Transactions On Intelligent Transportation Systems, Vol. 10, NO. 2, 2009

[46] Will Knight, Driverless Cars Are Further Away Than You Think, technologyreview.com, Featured Story In MIT Technology Review, 22 Oct 2013

[47] Chi-Ying Liang, Huei Peng, String Stability Analysis of Adaptive Cruise Controlled Vehicles, 2000 